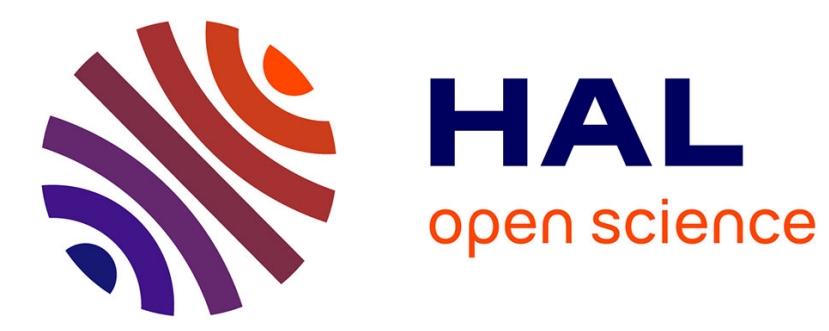

\title{
Chiral superstructures from homochiral Zn 2+, Co 2+, Fe 2+ -2,6-bis ( aryl ethylimine)pyridine complexes
}

Florina Dumitru, Arie van Der Lee, Mihail Barboiu

\section{To cite this version:}

Florina Dumitru, Arie van Der Lee, Mihail Barboiu. Chiral superstructures from homochiral Zn 2+ , Co 2+, Fe 2+ -2,6-bis ( aryl ethylimine)pyridine complexes. Chirality, 2019, 31 (10), pp.763-775. 10.1002/chir.23115. hal-03031043

\section{HAL Id: hal-03031043 \\ https://hal.umontpellier.fr/hal-03031043}

Submitted on 30 Nov 2020

HAL is a multi-disciplinary open access archive for the deposit and dissemination of scientific research documents, whether they are published or not. The documents may come from teaching and research institutions in France or abroad, or from public or private research centers.
L'archive ouverte pluridisciplinaire HAL, est destinée au dépôt et à la diffusion de documents scientifiques de niveau recherche, publiés ou non, émanant des établissements d'enseignement et de recherche français ou étrangers, des laboratoires publics ou privés. 


\section{Chiral superstructures from homochiral $\mathrm{Zn}^{2+}, \mathrm{Co}^{2+}, \mathrm{Fe}^{2+}-2,6$-bis(aryl ethylimine)pyridine complexes}

Abstract: We report hierarchical supramolecular organization of metallosupramolecular homochiral complexes 1- $\Lambda$ $(\mathrm{S}, \mathrm{S}, \mathrm{S}, \mathrm{S})-\mathrm{M}^{2+} / \mathbf{1 -}-\mathrm{-}-(\mathrm{R}, \mathrm{R}, \mathrm{R}, \mathrm{R})-\mathrm{M}^{2+}$ and $2-\Lambda-(\mathrm{S}, \mathrm{S}, \mathrm{S}, \mathrm{S})-\mathrm{M}^{2+} / \mathbf{2 -}$ $\Delta-(R, R, R, R)-M^{2+}$ of $\mathrm{M}^{2+}=\mathrm{Co}^{2+}, \mathrm{Fe}^{2+}, \mathrm{Zn}^{2+}$ metal ions with chiral pseudo-terpyridine-type ligands: $1-(S, S)$ or $1-(R, R)=2,6-$ bis(naphthyl ethylimine)pyridine and $2-(\mathrm{S}, \mathrm{S})$ or $2-(\mathrm{R}, \mathrm{R})=2,6-$ bis(phenyl-ethylimine)pyridine.
Circular dichroism measurements in solution were used to confirm the enantiomeric nature of all twelve complexes. For crystal structures of 1- $\Lambda$ - $(\mathrm{S}, \mathrm{S}, \mathrm{S}, \mathrm{S})-\mathrm{M}^{2+}$ or 1- $\Delta-(\mathrm{R}, \mathrm{R}, \mathrm{R}, \mathrm{R})-\mathrm{M}^{2+}$ complexes, absolute configurations $\{\Delta$ (or $\mathrm{P}), \Lambda$ (or $\mathrm{M})\}$ were confirmed by refinement of the Flack parameter $x$ : $-0.007 \leq x$ $\leq 0.11$ for the single-crystals of $1-\Lambda-(S, S, S, S)-M^{2+} / 1-\Delta-$ $(\mathrm{R}, \mathrm{R}, \mathrm{R}, \mathrm{R})-\mathrm{M}^{2+}, \mathbf{2}-\Lambda^{-}(\mathrm{S}, \mathrm{S}, \mathrm{S}, \mathrm{S})-\mathrm{Fe}^{2+}$ and $2-\Delta^{-}(\mathrm{R}, \mathrm{R}, \mathrm{R}, \mathrm{R})-\mathrm{Co}^{2+}$.

\section{Florina Dumitru, ${ }^{[a, b]}$ Arie van der Lee,${ }^{[a]}$ Mihail Barboiu ${ }^{*[a]}$}

Keywords: metallosupramolecular complexes, imine, self-assembly, aromatic interactions, chiral single-crystals

\section{Introduction}

Chiral symmetry breaking and transfer of chiral information from molecular toward supramolecular level through noncovalent interactions are topics of great interest. Molecular and supramolecular chirality may be both used as tools to assemble systems into dissymmetric crystalline architectures based on selective chiral packing. ${ }^{1}$ Chiral metallosupramolecular complexes are of considerable interest due to their important applications as stereodynamic probes for chiral sensing, ${ }^{2,3}$ for the preparation of chiral catalysts, ${ }^{4,5}$ and for the development of multifunctional materials. ${ }^{6}$ In the design and the synthesis of chiral ligands that, upon coordination with metal ions, can induce high stereoselectivity at a supramolecular level, Schiff bases with stereogenic centres in their backbones have been extensively used as powerful tools for the spontaneous generation of chiral superstructures. Typically, these chiral Schiff bases are obtained by condensation between aldehydes and chiral primary amines, and, among the most frequently used chiral amines, one can include enantiomeric pairs of R-(+)-1-/ S-(-)-1-phenylethylamine and $\mathrm{R}-(+)-1-/ \mathrm{S}-(-)-1$-naphthylethylamine. ${ }^{7-41}$

Herein, we report six enantiomeric pairs of $\mathrm{M}^{2+}=\mathrm{Zn}^{2+}, \mathrm{Co}^{2+}$, $\mathrm{Fe}^{2+}$ mononuclear complexes: 1- $\wedge-(\mathrm{S}, \mathrm{S}, \mathrm{S}, \mathrm{S})-\mathrm{M}^{2+} / \mathbf{1}-\Delta-(\mathrm{R}, \mathrm{R}, \mathrm{R}, \mathrm{R})-$ $\mathrm{M}^{2+}$ and 2- $\Lambda-(\mathrm{S}, \mathrm{S}, \mathrm{S}, \mathrm{S})-\mathrm{M}^{2+} / \mathbf{2}-\Delta-(\mathrm{R}, \mathrm{R}, \mathrm{R}, \mathrm{R})-\mathrm{M}^{2+}$, where 1-(S,S) or 1-(R,R) are 2,6-bis(naphthylethylimine)pyridine and 2-(S,S) or 2$(R, R)=2,6$-bis (phenylethylimine)pyridine, Schiff bases containing two stereogenic centers. The metal ions are used to template the Schiff base formation from R-(+)-1-/S-(-)-1-phenylethylamine, $\mathrm{R}-(+)-1-/ \mathrm{S}-(-)-1$-naphthylethylamine and 2,6pyridine-dicarbox-aldehyde (Scheme 1). The resulted pseudoterpyridines ligands orthogonally wrap around the metal ion centres, positioning their four stereogenic centers, such that the metal ions are overally surrounded by chiral coordination centres, as revealed by X-ray crystal structures and circular dichroism (CD) spectra.

The metal ion coordination by chiral molecular ligands results in the formation of highly compact chiral supramolecular homodimers: 1- $\Lambda-(\mathrm{S}, \mathrm{S}, \mathrm{S}, \mathrm{S})-\mathrm{M}^{2+}$ and 1-- $\Delta-(\mathrm{R}, \mathrm{R}, \mathrm{R}, \mathrm{R})-\mathrm{M}^{2+}$ stabilized by strong internal $\pi-\pi$ stacking interactions between lateral aromatic arms and central pyridine moiety. Further selfassembly in the resolved solid state homochiral metallosupramolecular domains is observed in some cases with the formation of unique double-stranded monohelices with single handedness. The solid-phase homochirality is determined by a subtle interplay of four directional orthogonal-pseudo-terpyridine coordination geometry and "locked" by weak interactions $\pi-\pi /$ $\mathrm{CH} \cdots \pi$ interactions between peripheral aryl rings. ${ }^{6,42-44}$

Usually, such helical metallosupramolecular complexes crystallize in distinct alternative $\mathrm{P}$ and $\mathrm{M}$ columns or layers of $\Delta$ or $\wedge$ mirror enantiomers, but overall the crystals are racemic. Intermolecular crystal packing is usually not discriminating: a system of enantiomeric complexes evolves towards solid-phase homochirality if homochiral interactions between molecules are more stable than heterochiral interactions, ${ }^{45}$ but the greater stability of homochiral versus heterochiral interactions is a necessary but not a sufficient condition for establishing solidphase homochirality.

There are few previous examples of direct crystallization of enantiopure helical supramolecular single crystals, ${ }^{46-48}$ more often the crystal is racemic since homochiral layers of opposite chirality could be present and connected via different chirality inverting interactions. Examples of homochiral supramolecular helices were reported by us, in our previous work $^{49}$ on complexes of $\mathrm{Zn}^{2+}, \mathrm{Co}^{2+}, \mathrm{Fe}^{2+}, \mathrm{Pb}^{2+}$ metal ions with bis(arene imine)pyridines ligands, but these examples are exclusively based on achiral ligands and the resulted solid state chirality is promoted by constitutional chiral affinity of supramolecular helices of the same handedness, interacting via their van der Waals hypersurfaces. ${ }^{49 a}$

[a] Institut Européen des Membranes, UMR-CNRS 5635, Université Montpellier, Place Eugène Bataillon, CC 047, 34095 Montpellier Cedex 5, France.

[b] University Politehnica of Bucharest, 1-7 Polizu st., 011061 , Bucharest, Romania.

Corresponding author: Mihail Barboiu, Institut Europeen des Membranes, Montpellier, France.

Email: mihail-dumitru.barboiu@umontpellier.fr

Received: ((will be filled in by the editorial staff)) Revised: ((will be filled in by the editorial staff)) 


\section{Materials and Methods}

R-(+)-1-/S-(-)-1-phenylethylamine, R-(+)-1-/S-(-)-1-naphthylethylamine, 2,6-pyridinemethanol, $\mathrm{MnO}_{2}, \mathrm{Zn}\left(\mathrm{CF}_{3} \mathrm{SO}_{3}\right)_{2}, \mathrm{Fe}\left(\mathrm{BF}_{4}\right)_{2}$ $\cdot 6 \mathrm{H}_{2} \mathrm{O}, \mathrm{Co}\left(\mathrm{BF}_{4}\right)_{2} \cdot 6 \mathrm{H}_{2} \mathrm{O}$, and $\mathrm{CD}_{3} \mathrm{CN}$ were purchased from Aldrich and used as received. All other reagents were obtained from commercial suppliers and used without further purification. All organic solutions were routinely dried over molecular sieves $4 \AA$. 2,6-pyridinedicarboxaldehyde was prepared by oxidation of 2,6pyridinemethanol with activated $\mathrm{MnO}_{2}$, according to the procedure described in the literature. ${ }^{50}$

${ }^{1} \mathrm{H}$-NMR spectra were recorded on DRX $400 \mathrm{MHz}$ Bruker Avance spectrometer, in $C_{3} \mathrm{CN}$, with the use of the residual solvent peak as reference. Mass spectrometric studies were performed in the positive ion mode using a quadrupole mass spectrometer (Micromass, Platform 2+). Samples were dissolved in acetonitrile and were continuously introduced into the mass spectrometer at a flow rate of $10 \mathrm{~mL} / \mathrm{min}$ through a Waters $616 \mathrm{HPLC}$ pump. The temperature $\left(80^{\circ} \mathrm{C}\right)$, the extraction cone voltage $\left(V_{c}=5-10 \mathrm{~V}\right)$ was usually set to avoid fragmentations. The notations used for the assignments of the ${ }^{1} \mathrm{H}$-NMR signals are given below.

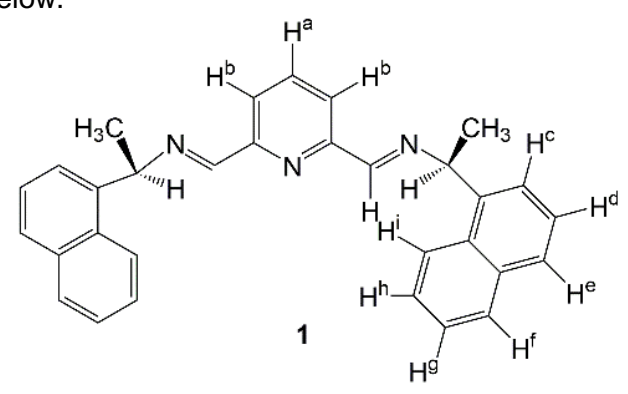

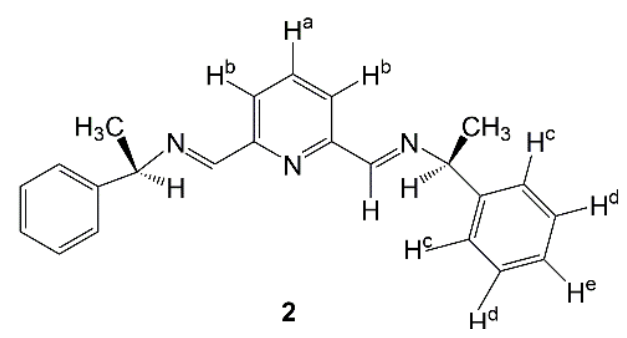

UV-vis absorbance spectra were recorded using a Kontron Instruments Uvikon 923 spectrometer, in acetonitrile $10^{-4}-10^{-5} \mathrm{M}$, with acetonitrile as a reference. $C D$ spectra were measured on a Jasco J-810 spectrometer, with a DC150 W xenon lamp. Measurements were collected using a $1 \mathrm{~mm}$ path-length quartz cuvette and the standard parameters used were: bandwidth 2 $\mathrm{nm}$, response time $1 \mathrm{~s}$, wavelength scan range 190-600 nm, data pitch $0.2 \mathrm{~nm}$, scanning speed $50 \mathrm{~nm} \cdot \mathrm{min}^{-1}$, and accumulation 5 .

X-ray Single Crystal Diffraction Structure solution and refinement. (Table 1) Crystal evaluation and data collection were performed on a Rigaku Oxford-Diffraction Xcalibur-I or a Gemini-S diffractometer with sealed-tube Mo-Ka radiation using the CrysAlis Pro program ${ }^{51}$. The same program was used for the integration of the data using default parameters, for the empirical absorption correction using spherical harmonics employing symmetry-equivalent and redundant data, and the correction for Lorentz and polarization effects. The crystal structures were solved using the ab-initio iterative charge flipping method with parameters described elsewhere ${ }^{52}$ using the Superflip program ${ }^{53}$ and they were refined using full-matrix leastsquares procedures as implemented in CRYSTALS ${ }^{54}$ on all independent reflections with $1>2 \sigma(I)$. Special attention was given to the determination of the absolute structure of each compound. The compounds 1-(R,R,R,R)- $\mathrm{Co}^{2+}$ and 1-(S,S,S,S) $\mathrm{Co}^{2+}$ crystallize each in an enantiomorphic space group. The structure solution was therefore done in each space group of the enantiomorphic pair and the space group was chosen on the basis of having the Flack parameter close to 0.00 . The other compounds crystallize in non-enantiomorphic Sohncke space groups and the structure was inverted if the Flack parameter was found to be close to 1.0. All final Flack and Hooft parameters ${ }^{55}$ are very close to 0.00 . Following an analysis based on maximum likelihood estimation and Bayesian statistics the chance having an enantiopure material is in all cases $100 \%{ }^{56}$ The $\mathrm{H}$ atoms were all located in a difference map, but repositioned geometrically. They were initially refined with soft restraints on the bond lengths and angles to regularize their geometry $(\mathrm{C}---\mathrm{H}$ in the range 0.93-0.98 $\AA$ ) and $U_{\text {iso }}(\mathrm{H})$ (in the range 1.2-1.5 times $U_{\text {eq }}$ of the parent atom), after which the positions were refined with riding constraints. ${ }^{57}$ In some cases thermal similarity restraints were used especially for solvent molecules. In one case an acetonitrile solvent molecule was refined as a rigid group. CCDC 1910805-1910812 contains the supplementary crystallographic data for this paper. These data can be obtained free of charge from The Cambridge Crystallographic Data Centre via www.ccdc.cam.ac.uk/structures

General procedure for the synthesis of homonuclear

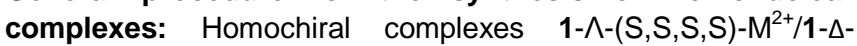
$(\mathrm{R}, \mathrm{R}, \mathrm{R}, \mathrm{R})-\mathrm{M}^{2+}$ and $2-\Lambda-(\mathrm{S}, \mathrm{S}, \mathrm{S}, \mathrm{S})-\mathrm{M}^{2+} / 2-\Delta-(\mathrm{R}, \mathrm{R}, \mathrm{R}, \mathrm{R})-\mathrm{M}^{2+} \mathrm{M}^{2+}=$ $\mathrm{Zn}^{2+}, \mathrm{Fe}^{2+}, \mathrm{Co}^{2+}$ have been obtained by template reaction between 2,6- pyridine-dicarboxaldehyde $(0.148 \mathrm{mmol})$ and corresponding chiral amines R-(+)-1- or S-(-)-1-phenylethylamine and $\mathrm{R}-(+)-1$ - or S-(-)-1-naphthyl-ethylamine in the presence of stoichiometric amounts of $\mathrm{Zn}\left(\mathrm{CF}_{3} \mathrm{SO}_{3}\right)_{2}, \mathrm{Fe}\left(\mathrm{BF}_{4}\right)_{2} \cdot 6 \mathrm{H}_{2} \mathrm{O}$, $\mathrm{Co}\left(\mathrm{BF}_{4}\right)_{2} \cdot 6 \mathrm{H}_{2} \mathrm{O}$, in acetonitrile, in the molar ratio of aldehyde:amine:metal salt $=1: 2: 0.5$. The reactions were performed typically on a $10 \mathrm{mg}$ scale of ligand $/ \mathrm{mL}$ solvent. The reactants were dissolved in $\mathrm{CD}_{3} \mathrm{CN}(1 \mathrm{~mL})$, and stirred overnight at $60^{\circ} \mathrm{C}$. These solutions were monitored by ${ }^{1} \mathrm{H}-\mathrm{NMR}$ and ESImass spectrometries. Layering the solutions of complexes in acetonitrile with isopropyl ether at room temperature resulted in a unique set of single crystals suitable for X-ray single-crystal experiments.

Complex 1- $\Delta-(\mathrm{R}, \mathrm{R}, \mathrm{R}, \mathrm{R})-\mathrm{Zn}^{2+}$. Yellow crystals. ${ }^{1} \mathrm{H}-\mathrm{RMN}(400$ $\left.\mathrm{MHz}, \mathrm{CD}_{3} \mathrm{CN}-\mathrm{d}_{3}, \delta\right) 8.22(\mathrm{~s}, 4 \mathrm{H} ; \mathrm{CH}=\mathrm{N}), 7.82-7.80(\mathrm{~d}, J=8 \mathrm{~Hz}$, $\left.4 \mathrm{H} ; \mathrm{H}^{\mathrm{b}}\right), 7.70-7.64\left(\mathrm{~m}, J=7.2 \mathrm{~Hz}, J=8 \mathrm{~Hz}, 10 \mathrm{H} ; \mathrm{H}^{\mathrm{a}}-\mathrm{H}^{\mathrm{i}}-\mathrm{H}^{\mathrm{f}}\right), 7.62-$ $7.60\left(\mathrm{t}, J=8 \mathrm{~Hz}, 4 \mathrm{H} ; \mathrm{H}^{\mathrm{h}}\right), 7.56-7.52\left(\mathrm{t}_{\mathrm{d}}, J=8 \mathrm{~Hz}, 4 \mathrm{H} ; \mathrm{H}^{\mathrm{g}}\right), 7.27-$ $7.25\left(\mathrm{~d}, J=7.6 \mathrm{~Hz}, 4 \mathrm{H} ; \mathrm{H}^{\mathrm{e}}\right), 7.07-7.03\left(\mathrm{t}, J=7.6 \mathrm{~Hz}, 4 \mathrm{H} ; \mathrm{H}^{\mathrm{d}}\right)$, $6.54-6.52\left(\mathrm{~d}, J=6.4 \mathrm{~Hz}, J=7.2 \mathrm{~Hz}, 4 \mathrm{H} ; \mathrm{H}^{\mathrm{c}}\right), 4.76-4.72(\mathrm{q}, J=$ $6.8 \mathrm{~Hz}, 4 \mathrm{H}$; - $\mathrm{NCH}-$ ), 1.04-1.03 (d, $J=6.4 \mathrm{~Hz}, 12 \mathrm{H}$; $\mathrm{CH}_{3}$ ). UV-vis (acetonitrile $\left.7.425 \cdot 10^{-5} \mathrm{M}\right): \lambda_{\max }=282 \mathrm{~nm}, 220 \mathrm{~nm}$; MS (ESI, m/z): $473.36 \quad(100) \quad\left[\mathrm{Zn}(\mathbf{1}-\Delta-(\mathrm{R}, \mathrm{R}, \mathrm{R}, \mathrm{R}))_{2}\right]^{2+}, \quad 1095.72 \quad\left[\mathrm{Zn}\left(\mathbf{1}-\Delta^{-}\right.\right.$ $\left.(\mathrm{R}, \mathrm{R}, \mathrm{R}, \mathrm{R}))_{2}\right]\left(\mathrm{CF}_{3} \mathrm{SO}_{3}\right)^{+}$.

Complex 1- $\Lambda-(\mathrm{S}, \mathrm{S}, \mathrm{S}, \mathrm{S})-\mathrm{Zn}^{2+}$. Yellow crystals. ${ }^{1} \mathrm{H}-\mathrm{RMN}(400 \mathrm{MHz}$ $\left.\mathrm{CD}_{3} \mathrm{CN}-d_{3}, \delta\right) 8.22(\mathrm{~s}, 4 \mathrm{H} ; \mathrm{CH}=\mathrm{N}), 7.82-7.80\left(\mathrm{~d}, J=8 \mathrm{~Hz}, 4 \mathrm{H} ; \mathrm{H}^{\mathrm{b}}\right)$, 7.70-7.64 (m, $\left.J=7.2 \mathrm{~Hz}, J=8 \mathrm{~Hz} 10 \mathrm{H}, \mathrm{H}^{\mathrm{a}}-\mathrm{H}^{\mathrm{i}}-\mathrm{H}^{\mathrm{f}}\right), 7.62-7.60$ (t, $J$ $\left.=8 \mathrm{~Hz}, 4 \mathrm{H} ; \mathrm{H}^{\mathrm{h}}\right), 7.56-7.52\left(\mathrm{t}_{\mathrm{d}}, J=8 \mathrm{~Hz}, 4 \mathrm{H} ; \mathrm{H}^{\mathrm{g}}\right), 7.27-7.25(\mathrm{~d}, J=$ $\left.7.6 \mathrm{~Hz}, 4 \mathrm{H}, \mathrm{H}^{\mathrm{e}}\right), 7.07-7.03\left(\mathrm{t}, J=7.6 \mathrm{~Hz}, 4 \mathrm{H}, \mathrm{H}^{\mathrm{d}}\right), 6.54-6.52(\mathrm{~d}, J$ $\left.=6.4 \mathrm{~Hz}, J=7.2 \mathrm{~Hz}, 4 \mathrm{H} ; \mathrm{H}^{\mathrm{c}}\right), 4.77-4.72(\mathrm{q}, J=6.8 \mathrm{~Hz}, 4 \mathrm{H}$; -

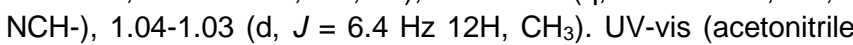
$\left.7.425 \cdot 10^{-5} \mathrm{M}\right): \lambda_{\max }=282 \mathrm{~nm}, 220 \mathrm{~nm}$; MS (ESI, m/z): 473.36 (100) $\quad\left[\mathrm{Zn}(\mathbf{1}-\Lambda-(\mathrm{S}, \mathrm{S}, \mathrm{S}, \mathrm{S}))_{2}\right]^{2+}, \quad 1095.78 \quad\left[\mathrm{Zn}\left(\mathbf{1}-\Lambda^{-}-\right.\right.$ $\left.(\mathrm{S}, \mathrm{S}, \mathrm{S}, \mathrm{S}))_{2}\right]\left(\mathrm{CF}_{3} \mathrm{SO}_{3}\right)^{+}$. 
TABLE 1 Crystallographic information on data collection and structure refinement.

\begin{tabular}{|c|c|c|c|c|c|c|c|c|}
\hline & $\begin{array}{c}1-\Delta-(R, R, R, R)- \\
C^{2+}\end{array}$ & $\begin{array}{c}1-\Lambda-(\mathrm{S}, \mathrm{S}, \mathrm{S}, \mathrm{S}) \\
\mathrm{Co}^{2+}\end{array}$ & $\begin{array}{c}1-\Delta-(R, R, R, R)- \\
F^{2+}\end{array}$ & $\begin{array}{c}1-\Lambda-(\mathrm{S}, \mathrm{S}, \mathrm{S}, \mathrm{S}) \\
\mathrm{Fe}^{2+}\end{array}$ & $\begin{array}{c}1-\Delta-(R, R, R, R)- \\
Z^{2+}\end{array}$ & $\begin{array}{c}1-\Lambda-(\mathrm{S}, \mathrm{S}, \mathrm{S}, \mathrm{S}) \\
\mathrm{Zn}^{2+}\end{array}$ & $\begin{array}{c}2-\Delta-(R, R, R, R)- \\
C^{2+}\end{array}$ & $\begin{array}{c}2-\Lambda-(\mathrm{S}, \mathrm{S}, \mathrm{S}, \mathrm{S}) \\
\mathrm{Fe}^{2+}\end{array}$ \\
\hline formula & $\begin{array}{c}\mathrm{C}_{62} \mathrm{H}_{54} \mathrm{~B}_{2} \mathrm{CoF}_{8} \mathrm{~N} \\
6\end{array}$ & $\begin{array}{c}\mathrm{C}_{62} \mathrm{H}_{54} \mathrm{~B}_{2} \mathrm{CoF}_{8} \mathrm{~N} \\
6\end{array}$ & $\begin{array}{c}\mathrm{C}_{66} \mathrm{H}_{60} \mathrm{~B}_{2} \mathrm{~F}_{8} \mathrm{FeN} \\
8\end{array}$ & $\begin{array}{c}\mathrm{C}_{66} \mathrm{H}_{60} \mathrm{~B}_{2} \mathrm{~F}_{8} \mathrm{Fe} \\
\mathrm{N}_{8}\end{array}$ & $\begin{array}{c}\mathrm{C}_{64} \mathrm{H}_{54} \mathrm{~F}_{6} \mathrm{~N}_{6} \mathrm{O}_{6} \mathrm{~S} \\
{ }_{2} \mathrm{Zn}\end{array}$ & $\begin{array}{c}\mathrm{C}_{64} \mathrm{H}_{54} \mathrm{~F}_{6} \mathrm{~N}_{6} \mathrm{O}_{6} \\
\mathrm{~S}_{2} \mathrm{Zn}\end{array}$ & $\begin{array}{c}\mathrm{C}_{48} \mathrm{H}_{49} \mathrm{~B}_{2} \mathrm{CoF}_{8} \mathrm{~N} \\
7\end{array}$ & $\mathrm{C}_{50} \mathrm{H}_{52} \mathrm{~B}_{2} \mathrm{~F}_{8} \mathrm{FeN}_{8}$ \\
\hline moiety & $\begin{array}{c}\mathrm{C}_{62} \mathrm{H}_{54} \mathrm{CoN}_{6}, 2( \\
\left.\mathrm{BF}_{4}\right)\end{array}$ & $\begin{array}{c}\mathrm{C}_{62} \mathrm{H}_{54} \mathrm{CoN}_{6}, 2(\mathrm{~B} \\
\left.\mathrm{F}_{4}\right)\end{array}$ & $\begin{array}{l}\mathrm{C}_{62} \mathrm{H}_{54} \mathrm{FeN}_{6}, 2( \\
\left.\mathrm{BF}_{4}\right), 2\left(\mathrm{C}_{2} \mathrm{H}_{3} \mathrm{~N}\right)\end{array}$ & $\begin{array}{c}\mathrm{C}_{62} \mathrm{H}_{54} \mathrm{FeN}_{6}, 2 \\
\left(\mathrm{BF}_{4}\right), 2\left(\mathrm{C}_{2} \mathrm{H}_{3}\right. \\
\mathrm{N})\end{array}$ & $\begin{array}{c}\mathrm{C}_{62} \mathrm{H}_{54} \mathrm{~N}_{6} \mathrm{Zn}, 2( \\
\left.\mathrm{CF}_{3} \mathrm{O}_{3} \mathrm{~S}\right)\end{array}$ & $\begin{array}{c}\mathrm{C}_{62} \mathrm{H}_{54} \mathrm{~N}_{6} \mathrm{Zn}, 2 \\
\left(\mathrm{CF}_{3} \mathrm{O}_{3} \mathrm{~S}\right)\end{array}$ & $\begin{array}{c}\mathrm{C}_{46} \mathrm{H}_{46} \mathrm{CoN}_{6}, 2(\mathrm{~B} \\
\left.\mathrm{F}_{4}\right), \mathrm{C}_{2} \mathrm{H}_{3} \mathrm{~N}\end{array}$ & $\begin{array}{c}\mathrm{C}_{46} \mathrm{H}_{46} \mathrm{FeN}_{6}, 2\left(\mathrm{BF}_{4}\right), \\
2\left(\mathrm{C}_{2} \mathrm{H}_{3} \mathrm{~N}\right)\end{array}$ \\
\hline$T(\mathrm{~K})$ & 175 & 175 & 175 & 175 & 175 & 175 & 175 & 175 \\
\hline $\begin{array}{c}\text { spacegroup } \\
\text { crystal } \\
\text { system }\end{array}$ & $\begin{array}{c}P 6_{5} 22 \\
\text { hexagonal }\end{array}$ & $\begin{array}{c}P 6,22 \\
\text { hexagonal }\end{array}$ & $\begin{array}{c}P 2_{1} 2_{1} 2_{1} \\
\text { orthorhombic }\end{array}$ & $\begin{array}{c}P 2_{1} 2_{1} 2_{1} \\
\text { orthorhombic }\end{array}$ & $\begin{array}{c}P 22_{1} 2_{1} \\
\text { orthorhombic }\end{array}$ & $\begin{array}{c}P 22_{1} 2_{1} \\
\text { orthorhombic }\end{array}$ & $\begin{array}{c}P 2_{1} 2_{1} 2_{1} \\
\text { orthorhombic }\end{array}$ & $\begin{array}{c}P 2_{1} 2_{1} 2_{1} \\
\text { orthorhombic }\end{array}$ \\
\hline$a(\AA)$ & $12.8190(14)$ & $12.8233(3)$ & $12.68634(16)$ & $12.6784(2)$ & $13.3143(2)$ & $13.3163(2)$ & $11.1126(8)$ & $12.7169(5)$ \\
\hline$b(\AA)$ & $12.8190(14)$ & $12.8233(3)$ & $20.5481(3)$ & $20.5187(4)$ & $19.8115(4)$ & $19.8111(4)$ & $12.9994(9)$ & $17.9704(8)$ \\
\hline$c(\AA)$ & $59.951(3)$ & $59.9965(15)$ & $21.7836(3)$ & $21.7822(4)$ & $21.1578(5)$ & $21.1606(6)$ & $31.6636(18)$ & $20.5289(10)$ \\
\hline 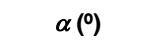 & 90 & 90 & 90 & 90 & 90 & 90 & 90 & 90 \\
\hline$\beta($ () & 90 & 90 & 90 & 90 & 90 & 90 & 90 & 90 \\
\hline$\gamma\left({ }^{\circ}\right)$ & 120 & 120 & 90 & 90 & 90 & 90 & 90 & 90 \\
\hline$V\left(\hat{\AA}^{3}\right)$ & $8531.7(5)$ & $8543.9(2)$ & $5678.55(8)$ & $5666.52(11)$ & $5580.92(13)$ & $5582.39(16)$ & $4574.0(3)$ & $4691.4(2)$ \\
\hline z & 6 & 6 & 4 & 4 & 4 & 4 & 4 & 4 \\
\hline$\rho\left(\mathrm{gcm}^{-3}\right)$ & 1.303 & 1.301 & 1.397 & 1.400 & 1.484 & 1.483 & 1.389 & 1.408 \\
\hline$M_{\mathrm{r}}\left(\mathrm{gmol}^{-1}\right)$ & 1115.66 & 1115.66 & 1194.69 & 1194.69 & 1246.64 & 1246.64 & 956.49 & 994.47 \\
\hline$\mu\left(\mathrm{mm}^{-1}\right)$ & 0.373 & 0.372 & 0.343 & 0.344 & 0.596 & 0.596 & 0.451 & 0.399 \\
\hline $\boldsymbol{R}_{\text {int }}$ & 0.071 & 0.094 & 0.043 & 0.080 & 0.103 & 0.046 & 0.139 & 0.166 \\
\hline$\Theta_{\max }(\stackrel{0}{)})$ & 33.593 & 26.371 & 35.628 & 32.562 & 33.606 & 32.394 & 26.497 & 26.372 \\
\hline $\begin{array}{l}\text { resolution } \\
\text { (A) }\end{array}$ & 0.68 & 0.80 & 0.64 & 0.66 & 0.80 & 0.66 & 0.80 & 0.83 \\
\hline $\begin{array}{c}N_{\text {tot }} \\
\text { (measured) }\end{array}$ & 68681 & 44040 & 127786 & 110475 & 46088 & 108276 & 69896 & 71765 \\
\hline$N_{\text {ref }}$ (unique) & 21031 & 5791 & 21371 & 18685 & 19522 & 18278 & 9440 & 9602 \\
\hline$N_{\text {ref }}(>2 \sigma(n))$ & 6102 & 4848 & 17959 & 13252 & 8854 & 14538 & 6334 & 5953 \\
\hline $\begin{array}{c}N_{\text {ref }} \text { (least- } \\
\text { squares) }\end{array}$ & 6102 & 4848 & 17959 & 13252 & 8854 & 14538 & 6334 & 5953 \\
\hline$N_{\text {par }}$ & 409 & 409 & 767 & 767 & 767 & 767 & 578 & 623 \\
\hline$<\sigma(I) / l>$ & 0.1005 & 0.1344 & 0.0588 & 0.1162 & 0.1845 & 0.0763 & 0.1888 & 0.2220 \\
\hline$R_{1}(\triangleright 2 \sigma(I))$ & 0.0733 & 0.0897 & 0.0634 & 0.0672 & 0.0742 & 0.0433 & 0.0671 & 0.0858 \\
\hline$w R_{2}(>2 \sigma(I))$ & 0.0580 & 0.0752 & 0.0678 & 0.0525 & 0.0497 & 0.0431 & 0.0475 & 0.0800 \\
\hline$R_{1}$ (all) & 0.1940 & 0.1058 & 0.0774 & 0.1065 & 0.1779 & 0.0639 & 0.1097 & 0.1412 \\
\hline$w R_{2}$ (all) & 0.0734 & 0.0825 & 0.0802 & 0.0550 & 0.0959 & 0.0443 & 0.0663 & 0.1202 \\
\hline GOF & 1.0436 & 1.0510 & 1.0756 & 1.1828 & 1.0631 & 1.0657 & 1.1902 & 1.1287 \\
\hline$\Delta \rho\left(\mathrm{e}^{-3}\right)$ & $-0.88 / 1.38$ & $-0.83 / 0.72$ & $-1.11 / 2.26$ & $-0.96 / 1.48$ & $-0.88 / 1.18$ & $-0.57 / 0.56$ & $-0.54 / 0.95$ & $-0.51 / 0.69$ \\
\hline $\begin{array}{l}\text { crystal size } \\
\left(\mathrm{mm}^{3}\right)\end{array}$ & $0.09 \times 0.15 \times 0.31$ & $0.07 \times 0.24 \times 0.24$ & $0.08 \times 0.23 \times 0.35$ & $\begin{array}{c}0.05 \times 0.15 \times 0 \\
30\end{array}$ & $0.08 \times 0.12 \times 0.23$ & $\begin{array}{c}0.15 \times 0.20 \times 0 \\
23\end{array}$ & $0.12 \times 0.15 \times 0.22$ & $0.05 \times 0.12 \times 0.21$ \\
\hline $\begin{array}{c}\text { Flack } \\
\text { parameter }\end{array}$ & $0.04(3)$ & $0.11(3)$ & $0.01(1)$ & $0.02(2)$ & $0.02(1)$ & $-0.007(6)$ & $0.00(1)$ & $0.05(4)$ \\
\hline $\begin{array}{c}\text { Hooft } \\
\text { parameter }\end{array}$ & $0.03(2)$ & $0.01(1)$ & $-0.002(4)$ & $-0.008(6)$ & $0.008(6)$ & $-0.0125(1)$ & $-0.01(1)$ & $0.009(9)$ \\
\hline $\begin{array}{l}\text { Chiral } \\
\text { centers }\end{array}$ & $\Delta$-RRRR & $\Lambda$-SSSS & $\Delta$-RRRR & $\Lambda$-SSSS & $\Delta$-RRRR & $\Lambda$-SSSS & $\Delta$-RRRR & $\Lambda$-SSSS \\
\hline
\end{tabular}

Complex 1- $\Delta-(\mathrm{R}, \mathrm{R}, \mathrm{R}, \mathrm{R})-\mathrm{Fe}^{2+}$. Violet crystals. UV-vis (acetonitrile $\left.7.425 \cdot 10^{-5} \mathrm{M}\right): \lambda_{\max }=281 \mathrm{~nm}\left(35.57 \mathrm{~cm}^{-1}\right.$, MLCT d- $\left.\pi^{\star}\right), 484 \mathrm{~nm}$ $\left(20.67 \mathrm{~cm}^{-1}\right), 608 \mathrm{~nm}\left(16.45 \mathrm{~cm}^{-1}\right), 705 \mathrm{~nm}\left(14.2 \mathrm{~cm}^{-1}\right)$. MS (ESI, $m / z): 469.33(100) \quad\left[\mathrm{Fe}(1-\Delta-(\mathrm{R}, \mathrm{R}, \mathrm{R}, \mathrm{R}))_{2}\right]^{2+}, 1025.75 \quad[\mathrm{Fe}(\mathbf{1}-\Delta-$ $\left.(\mathrm{R}, \mathrm{R}, \mathrm{R}, \mathrm{R}))_{2}\right]\left(\mathrm{BF}_{4}\right)^{+}$.

Complex 1-^-(S,S,S,S)-Fe ${ }^{2+}$. Violet crystals. UV-vis (acetonitrile $\left.7.425 \cdot 10^{-5} \mathrm{M}\right): \lambda_{\max }=281 \mathrm{~nm}\left(35.57 \mathrm{~cm}^{-1}\right.$, MLCT d- $\left.\pi^{\star}\right), 484 \mathrm{~nm}$ $\left(20.67 \mathrm{~cm}^{-1}\right), 608 \mathrm{~nm}\left(16.45 \mathrm{~cm}^{-1}\right) . \mathrm{MS}(\mathrm{ESI}, \mathrm{m} / \mathrm{z}): 469.46$ (100) $\left[\mathrm{Fe}(\mathbf{1}-\wedge-(\mathrm{S}, \mathrm{S}, \mathrm{S}, \mathrm{S}))_{2}\right]^{2+}, 1025.88\left[\mathrm{Fe}(\mathbf{1}-\wedge-(\mathrm{S}, \mathrm{S}, \mathrm{S}, \mathrm{S}))_{2}\right]\left(\mathrm{BF}_{4}\right)^{+}$.

Complex 1- $\Delta-(R, R, R, R)-C^{2+}$. Brown crystals.UV-vis (acetonitrile $\left.7.425 \cdot 10^{-5} \mathrm{M}\right): \lambda_{\max }=281 \mathrm{~nm}\left(35.57 \mathrm{~cm}^{-1}, \mathrm{MLCT} \mathrm{d}-\pi^{\star}\right)$. MS (ESI, $m / z): 470.88(100)\left[\mathrm{Co}(1-\Delta-(\mathrm{R}, \mathrm{R}, \mathrm{R}, \mathrm{R}))_{2}\right]^{2+}, 1028.78$ [Co(1- $\Delta-$ $\left.(\mathrm{R}, \mathrm{R}, \mathrm{R}, \mathrm{R}))_{2}\right]\left(\mathrm{BF}_{4}\right)^{+}$. 
Complex 1-^-(S,S,S,S)-Co ${ }^{2+}$. Brown crystals. UV-vis (acetonitrile $\left.7.425 \cdot 10^{-5} \mathrm{M}\right): \lambda_{\max }=281 \mathrm{~nm}\left(35.57 \mathrm{~cm}^{-1}\right.$, MLCT d$\left.\pi^{\star}\right)$. MS (ESI, $\left.m / z\right): 470.88(100)\left[\mathrm{Co}(1-\wedge-(\mathrm{S}, \mathrm{S}, \mathrm{S}, \mathrm{S}))_{2}\right]^{2+}, 1028.78$ $\left[\mathrm{Co}(1-\Lambda-(\mathrm{S}, \mathrm{S}, \mathrm{S}, \mathrm{S}))_{2}\right]\left(\mathrm{BF}_{4}\right)^{+}$.

Complex 2- $\Delta-(\mathrm{R}, \mathrm{R}, \mathrm{R}, \mathrm{R})-\mathrm{Zn}^{2+}$. Yellow crystals. ${ }^{1} \mathrm{H}-\mathrm{RMN}(400$ $\left.\mathrm{MHz}, \mathrm{CD}_{3} \mathrm{CN}-d_{3}, \delta\right)$ 8.56-8.52 (t, $\left.J=7.6 \mathrm{~Hz}, J=8 \mathrm{~Hz}, 2 \mathrm{H} ; \mathrm{H}^{\mathrm{a}}\right)$, $8.30(\mathrm{~s}, 4 \mathrm{H} ; \mathrm{CH}=\mathrm{N}), 8.05-8.03\left(\mathrm{~d}, J=8 \mathrm{~Hz}, 4 \mathrm{H} ; \mathrm{H}^{\mathrm{b}}\right), 7.20-7.16(\mathrm{t}$, $J=7.2 \mathrm{~Hz}, J=7.6 \mathrm{~Hz}, 4 \mathrm{H} ; \mathrm{H}^{\mathrm{e}}$ ), 7.06-7.02 (t, $J=8 \mathrm{~Hz}, \mathrm{~J}=7.6 \mathrm{~Hz}$, $8 \mathrm{H} ; \mathrm{H}^{\mathrm{c}}$ ), 6.59-6.57 (d, $J=7.2 \mathrm{~Hz}, 8 \mathrm{H}, \mathrm{H}^{\mathrm{d}}$ ), 4.37-4.32 (q, $J=6.8$ $\mathrm{Hz}, 4 \mathrm{H} ; \mathrm{NCH}-$ ), 1.14-1.13 (d, $J=6.8 \mathrm{~Hz}, 12 \mathrm{H} ; \mathrm{CH}_{3}$ ). UV-vis (acetonitrile $7.425 \cdot 10^{-5} \mathrm{M}$ ): $\lambda_{\max }=317 \mathrm{~nm}, 210 \mathrm{~nm}$; MS (ESI, $\mathrm{m} / \mathrm{z}): \quad 373.24 \quad(100) \quad\left[\mathrm{Zn}(2-\Delta-(\mathrm{R}, \mathrm{R}, \mathrm{R}, \mathrm{R}))_{2}\right]^{2+}, \quad 895.55 \quad\left[\mathrm{Zn}\left(2-\Delta^{-}\right.\right.$ $\left.(\mathrm{R}, \mathrm{R}, \mathrm{R}, \mathrm{R}))_{2}\right]\left(\mathrm{CF}_{3} \mathrm{SO}_{3}\right)^{+}$.

Complex 2- $\wedge-(\mathrm{S}, \mathrm{S}, \mathrm{S}, \mathrm{S})-\mathrm{Zn}^{2+}$. Yellow crystals. ${ }^{1} \mathrm{H}-\mathrm{RMN}(400$ $\left.\mathrm{MHz}, \mathrm{CD}_{3} \mathrm{CN}-d_{3}, \delta\right) 8.56-8.52\left(\mathrm{t}, J=7.6 \mathrm{~Hz}, J=8 \mathrm{~Hz}, 2 \mathrm{H} ; \mathrm{H}^{\mathrm{a}}\right)$, $8.30(\mathrm{~s}, 4 \mathrm{H} ; \mathrm{CH}=\mathrm{N}), 8.05-8.03\left(\mathrm{~d}, J=8 \mathrm{~Hz}, 4 \mathrm{H} ; \mathrm{H}^{\mathrm{b}}\right), 7.20-7.16(\mathrm{t}$, $J=7.2 \mathrm{~Hz}, J=7.6 \mathrm{~Hz}, 4 \mathrm{H} ; \mathrm{H}^{\mathrm{e}}$ ), 7.06-7.02 (t, $J=8 \mathrm{~Hz}, \mathrm{~J}=7.6 \mathrm{~Hz}$, $\left.8 \mathrm{H} ; \mathrm{H}^{\mathrm{c}}\right), 6.59-6.57\left(\mathrm{~d}, J=7.2 \mathrm{~Hz}, 8 \mathrm{H}, \mathrm{H}^{\mathrm{d}}\right), 4.37-4.32(\mathrm{q}, J=6.8$ $\mathrm{Hz}, 4 \mathrm{H} ; \mathrm{NCH}-)$, 1.14-1.13 (d, $J=6.8 \mathrm{~Hz}, 12 \mathrm{H} ; \mathrm{CH}_{3}$ ). UV-vis (acetonitrile $7.425 \cdot 10^{-5} \mathrm{M}$ ): $\lambda_{\max }=318 \mathrm{~nm}, 210 \mathrm{~nm}$; MS (ESI, $\mathrm{m} / \mathrm{z}): \quad 373.24(100) \quad\left[\mathrm{Zn}(2-\wedge-(\mathrm{S}, \mathrm{S}, \mathrm{S}, \mathrm{S}))_{2}\right]^{2+}, 895.69 \quad[\mathrm{Zn}(2-\wedge-$ $\left.(\mathrm{S}, \mathrm{S}, \mathrm{S}, \mathrm{S}))_{2}\right]\left(\mathrm{CF}_{3} \mathrm{SO}_{3}\right)^{+}$.

Complex 2- $\Delta-(\mathrm{R}, \mathrm{R}, \mathrm{R}, \mathrm{R})-\mathrm{Fe}^{2+}$. Violet crystals.UV-vis (acetonitrile $\left.7.425 \cdot 10^{-5} \mathrm{M}\right): \lambda_{\max }=325 \mathrm{~nm}\left(30.76 \mathrm{~cm}^{-1}\right.$, MLCT d- $\left.\pi^{\star}\right), 480 \mathrm{~nm}$ $\left(20.83 \mathrm{~cm}^{-1}\right), 603 \mathrm{~nm}\left(16.58 \mathrm{~cm}^{-1}\right), 709 \mathrm{~nm}\left(14.09 \mathrm{~cm}^{-1}\right)$. MS (ESI, $\mathrm{m} / \mathrm{z}): 369.27(100) \quad\left[\mathrm{Fe}(2-\Delta-(\mathrm{R}, \mathrm{R}, \mathrm{R}, \mathrm{R}))_{2}\right]^{2+}, 825.67 \quad[\mathrm{Fe}(2-\Delta-$ $\left.(\mathrm{R}, \mathrm{R}, \mathrm{R}, \mathrm{R}))_{2}\right]\left(\mathrm{BF}_{4}\right)^{+}$.

Complex 2- $\wedge-(\mathrm{S}, \mathrm{S}, \mathrm{S}, \mathrm{S})-\mathrm{Fe}^{2+}$. Violet crystals. UV-vis (acetonitrile $\left.7.425 \cdot 10^{-5} \mathrm{M}\right): \lambda_{\max }=325 \mathrm{~nm}\left(30.76 \mathrm{~cm}^{-1}\right.$, MLCT d- $\left.\pi^{\star}\right), 480 \mathrm{~nm}$ $\left(20.83 \mathrm{~cm}^{-1}\right), 603 \mathrm{~nm}\left(16.58 \mathrm{~cm}^{-1}\right), 709 \mathrm{~nm}\left(14.09 \mathrm{~cm}^{-1}\right)$. MS (ESI, $\mathrm{m} / \mathrm{z}): 369.27(100) \quad\left[\mathrm{Fe}(2-\Lambda-(\mathrm{S}, \mathrm{S}, \mathrm{S}, \mathrm{S}))_{2}\right]^{2+}, 825.67 \quad\left[\mathrm{Fe}\left(2-\Lambda^{-}\right.\right.$ $\left.(\mathrm{S}, \mathrm{S}, \mathrm{S}, \mathrm{S}))_{2}\right]\left(\mathrm{BF}_{4}\right)^{+}$.

Complex 2- $\Delta-(\mathrm{R}, \mathrm{R}, \mathrm{R}, \mathrm{R})-\mathrm{Co}^{2+}$. Brown crystals. UV-vis (acetonitrile $\left.7.425 \cdot 10^{-5} \mathrm{M}\right): \lambda_{\max }=300 \mathrm{~nm}\left(33.32 \mathrm{~cm}^{-1}\right.$, MLCT d- $\left.\pi^{*}\right)$. MS (ESI, $m / z): 370.76(100) \quad\left[\mathrm{Co}(2-\Delta-(\mathrm{R}, \mathrm{R}, \mathrm{R}, \mathrm{R}))_{2}\right]^{2+}, 828.46 \quad[\mathrm{Co}(2-\Delta-$ $\left.(\mathrm{R}, \mathrm{R}, \mathrm{R}, \mathrm{R}))_{2}\right]\left(\mathrm{BF}_{4}\right)^{+}$.

Complex 2-^-(S,S,S,S)-Co $\mathrm{Co}^{2+}$ Brown crystals. UV-vis (acetonitrile $\left.7.425 \cdot 10^{-5} \mathrm{M}\right): \lambda_{\max }=302 \mathrm{~nm}\left(33.06 \mathrm{~cm}^{-1}\right.$, MLCT d- $\left.\pi^{*}\right)$. MS (ESI, m/z): $370.76(100)\left[\mathrm{Co}(2-\wedge-(\mathrm{S}, \mathrm{S}, \mathrm{S}, \mathrm{S}))_{2}\right]^{2+}, 828.77$ $\left[\mathrm{Co}(2-\wedge-(\mathrm{S}, \mathrm{S}, \mathrm{S}, \mathrm{S}))_{2}\right]\left(\mathrm{BF}_{4}\right)^{+}$.

\section{Results and Discussion}

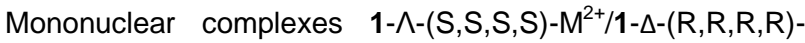
$\mathrm{M}^{2+}$ and $2-\wedge-(\mathrm{S}, \mathrm{S}, \mathrm{S}, \mathrm{S})-\mathrm{M}^{2+} / 2-\Delta-(\mathrm{R}, \mathrm{R}, \mathrm{R}, \mathrm{R})-\mathrm{M}^{2+}$ were obtained via template reactions, in the concentration range of $10 \mathrm{mg}$ ligand / $1 \mathrm{~mL}$ acetonitrile, from 2,6-pyridinedicarboxaldehyde (1 eq), R-(+)-1-/S-(-)-1-phenylethylamine and R-(+)-1-/S-()-1-naphthyl ethylamine (2 eq) and the corresponding metal ions, $\mathrm{Zn}^{2+}, \mathrm{Fe}^{2+}, \mathrm{Co}^{2+}(0.5 \mathrm{eq})$. The $\mathrm{Zn}^{2+}, \mathrm{Fe}^{2+}, \mathrm{Co}^{2+}$ metal ions used to obtain complexes with in situ generated bis(arylethylimine)pyridine ligands $\mathbf{1}$ and $\mathbf{2}$ are ions that prefer octahedral coordination geometry and determine the orthogonal orientation of the ligands similar to that found in the mononuclear metal complexes with the terpyridine ligands. The octahedral complexes of the $\mathrm{Zn}^{2+}, \mathrm{Fe}^{2+}$ and $\mathrm{Co}^{2+}$ ions are labile, suffer rapid equilibria in solution, and their stability can be correlated with their electronic configuration. On the other hand, the Schiff bases, bis(aryfimine)pyridine ligands $\mathbf{1}$ and 2, are strong field generators, $\pi$-acceptors, with low energy antibonding orbitals $\left(\right.$ LUMO, $\left.\pi^{\star}\right)$ and they form complexes with metal ions stabilized by charge transfer interactions metal(d)-to-ligand $\left(\pi^{\star}\right)$ MLCT.

NMR spectroscopy: Only $\mathrm{Zn}^{2+}$ ions, $d^{10}$ configuration, form diamagnetic complexes that can be characterized by ${ }^{1} \mathrm{H}$-NMR spectroscopy. The ${ }^{1} \mathrm{H}-\mathrm{NMR}$ spectra of the $1-\Lambda-(\mathrm{S}, \mathrm{S}, \mathrm{S}, \mathrm{S})-\mathrm{Zn}^{2+} / \mathbf{1}-$ $\Delta-(\mathrm{R}, \mathrm{R}, \mathrm{R}, \mathrm{R})-\mathrm{Zn}^{2+}$ and $2-\wedge-(\mathrm{S}, \mathrm{S}, \mathrm{S}, \mathrm{S})-\mathrm{Zn}^{2+} / \mathbf{2}-\Delta-(\mathrm{R}, \mathrm{R}, \mathrm{R}, \mathrm{R})-\mathrm{Zn}^{2+}$ homonuclear complexes (Fig. 1S-2S, Supporting Information) consist of a series of well-defined peaks characteristic to the $1: 2$ symmetric metal-ligand complex. In these spectra the $\mathrm{H}^{\mathrm{a}}$ pyridine protons signals of the ligand appear shifted to the weaker magnetic field than the signals corresponding to these protons in similar free bis(ary/lmine) pyridine ligands. ${ }^{49}$ Tridentate metal ion coordination determine the ligands to adopt during the template synthesis a cisoid conformation, corresponding to a terpyridine (terpy) type coordination site. The conversion of the all-transoid conformer into the energetically disfavored all-cisoid one upon metal complexation occurs at the cost of conformational energy, which is overcompensated by the interaction energy resulting from metal ion binding. ${ }^{49}$

b)

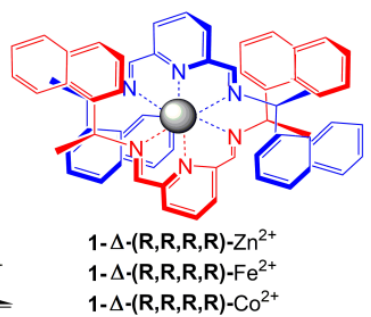<smiles>CC(NCc1cccc(CNC(C)C(C)c2cccc3ccccc23)n1)c1ccccc1</smiles>
$1, R, R$
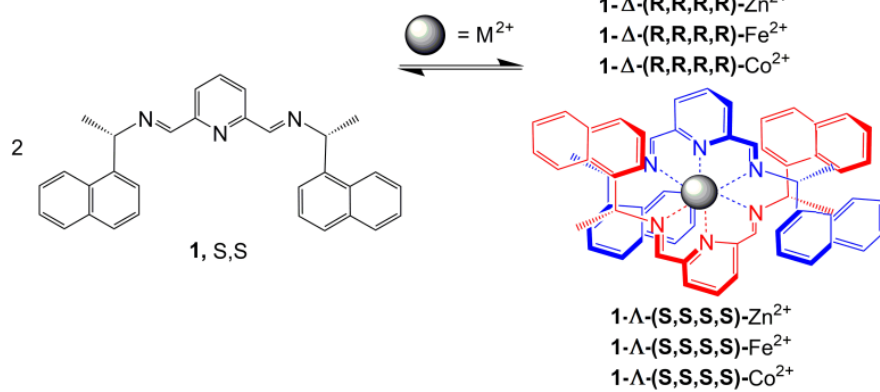
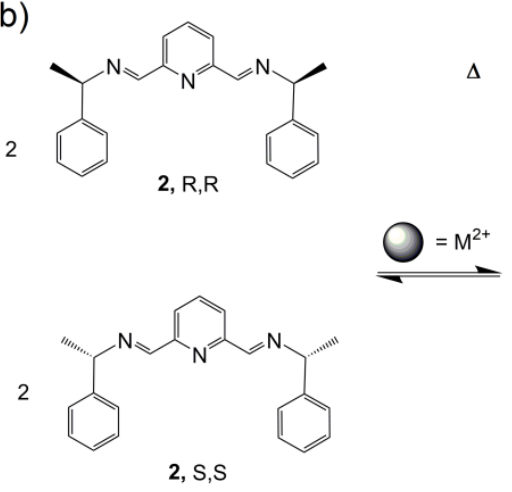

$\Delta$

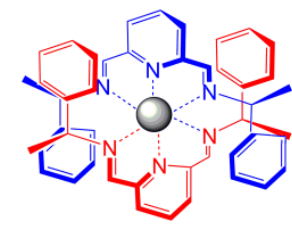

2- $\Delta \cdot(\mathbf{R}, \mathbf{R}, \mathbf{R}, \mathbf{R})-\mathrm{Zn}^{2+}$ 2- $\Delta-(R, R, R, R)-\mathrm{Fe}^{2+}$ $2-\Delta-(R, R, R, R)-C^{2+}$

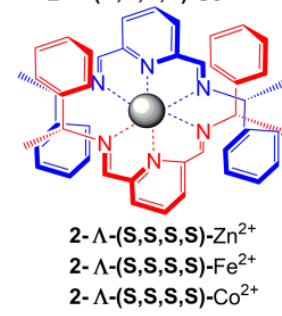

Scheme 1. One-pot synthesis of homochiral complexes: 1- - $-(S, S, S, S)-M^{2+} / 1-\Delta-(R, R, R, R)-M^{2+}$ and $2-\Lambda-(S, S, S, S)-M^{2+} / 2-\Delta-(R, R, R, R)-M^{2+} ; M^{2+}=Z^{2+}, F e^{2+}, C 0^{2+}$ 
ESI-MS spectrometry. Electrospray ionisation conditions (T $=80^{\circ} \mathrm{C}$, extraction cone voltage $\mathrm{V}_{\mathrm{c}}=10 \mathrm{~V}, 100 \%$ acetonitrile) were set to avoid the fragmentation (dissociation) of the complexes. ESI-mass spectra of complexes in acetonitrile solutions $\left(10^{-4} \mathrm{M}\right)$ showed the formation of double charged complex ions of $\left[M(1-\Delta-(R, R, R, R))_{2}\right]^{2+} /\left[M(1-\Lambda-(S, S, S, S))_{2}\right]^{2+}$ and $\left[\mathrm{M}(2-\Delta-(\mathrm{R}, \mathrm{R}, \mathrm{R}, \mathrm{R}))_{2}\right]^{2+} /\left[\mathrm{M}(2-\Lambda-(\mathrm{S}, \mathrm{S}, \mathrm{S}, \mathrm{S}))_{2}\right]^{2+}$ at $\mathrm{MW} / 2$ for all metal ions $\left(\mathrm{Zn}^{2+}, \mathrm{Fe}^{2+}, \mathrm{Co}^{2+}\right)$. The primary coordination sphere for metal ions $\left(\mathrm{CN}=6,\left[\mathrm{MN}_{6}\right]\right)$ remains intact in the solution; no substitution reactions with solvent molecules or anions present in the solution occur.

Electronic spectra. The information provided by the mass spectra, that in solution are present only the $[\mathrm{M}(1-\Delta-$ $\left.(\mathrm{R}, \mathrm{R}, \mathrm{R}, \mathrm{R}))_{2}\right]^{2+} / \quad\left[\mathrm{M}(\mathbf{1}-\mathrm{\Lambda}-(\mathrm{S}, \mathrm{S}, \mathrm{S}, \mathrm{S}))_{2}\right]^{2+}$ and $\left[\mathrm{M}\left(\mathbf{2}-\Delta^{-}\right.\right.$ $\left.(\mathrm{R}, \mathrm{R}, \mathrm{R}, \mathrm{R}))_{2}\right]^{2+} /\left[\mathrm{M}(2-\Lambda-(\mathrm{S}, \mathrm{S}, \mathrm{S}, \mathrm{S}))_{2}\right]^{2+}$ complexes, namely the primary coordination sphere of the metal ion is formed exclusively from the nitrogen atoms of the tridentate ligands, allow assignment of the $d$ - $d$ transitions for $\mathrm{Fe}^{2+}$ and $\mathrm{Co}^{2+}$ complexes (Table 2) from electronic UV-Vis spectra in solution. In octahedral coordination geometry, $\mathrm{Fe}^{2+}$ metal ions can present two spin states, depending on the strength of the ligand field, with the fundamental spectral terms: ${ }^{1} \mathrm{~A}_{1 \mathrm{~g}}$ (LS) and ${ }^{5} \mathrm{~T}_{2 \mathrm{~g}}$ (HS). For the $\mathrm{Co}^{2+}$ metal ion, in octahedral symmetry, the fundamental spectral terms are ${ }^{2} E(L S)$ and ${ }^{4} T_{1 g}(H S)$.

The UV-Vis spectra of the 1- $\Lambda$ - $(S, S, S, S)-F e^{2+} / 1-\Delta-(R, R, R, R)-$ $\mathrm{Fe}^{2+}$ and 2- $\Lambda$-(S,S,S,S)-Fe ${ }^{2+} / \mathbf{2}-\Delta-(\mathrm{R}, \mathrm{R}, \mathrm{R}, \mathrm{R})-\mathrm{Fe}^{2+}$ complexes have absorption maxima specific to the low-spin (LS) distorted octahedral geometries for $\mathrm{Fe}^{2+}$ ions. In these spectra, ${ }^{1} \mathrm{~A}_{1 \mathrm{~g}} \rightarrow$ ${ }^{1} \mathrm{~T}_{1 \mathrm{~g}}$ and ${ }^{1} \mathrm{~A}_{1 \mathrm{~g}} \rightarrow{ }^{1} \mathrm{~T}_{2 \mathrm{~g}}$ are assigned to the spin-allowed transitions. The weaker bands at $608 \mathrm{~nm}, 705 \mathrm{~nm}$ were assigned to spin forbidden transitions to triplet terms $\left({ }^{3} \mathrm{~T}_{1 \mathrm{~g}},{ }^{3} \mathrm{~T}^{2 \mathrm{~g}}\right)$. The bis(arylethylimino)pyridine ligands, $\mathbf{1}$ and 2, are strong field generators, $\pi$ acceptors, with antibonding orbitals (LUMO, $\pi^{\star}$ ) low in energy, and they form complexes stabilized by metal-toligand interactions (MLCT). MLCT bands for octahedral $\mathrm{Fe}^{2+}$ ion, $d^{6}$ occur in the low-energy domain $\sim 30 \mathrm{kK}$ and are very
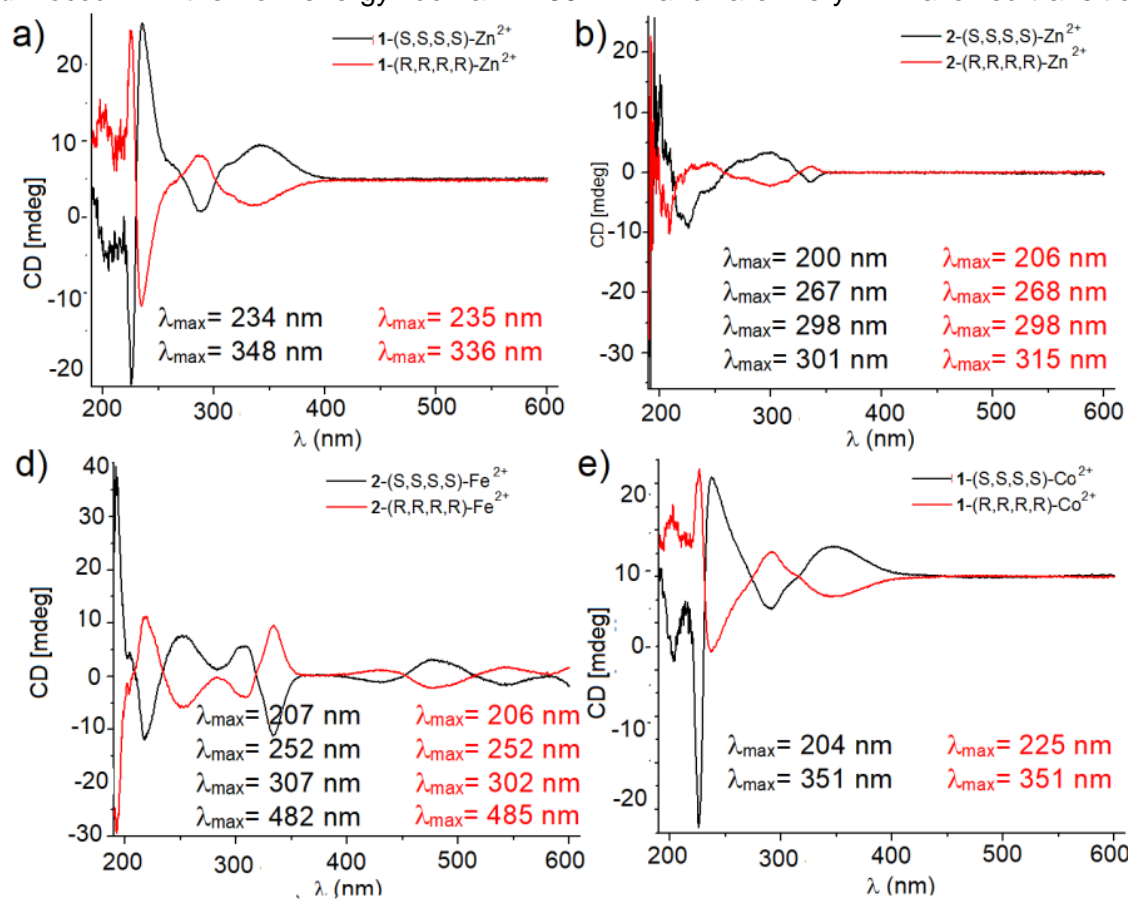

e)

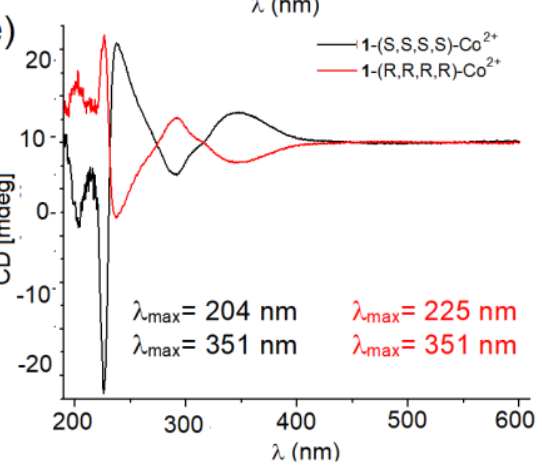

characteristic to the red-violet LS complexes of $\mathrm{Fe}^{2+}$ with ligands similar to bis(arylethylimino)pyridines: bypiridine (bpy) and phenanthroline (1,10-phen), $\left.\left[\mathrm{Fe}(\mathrm{bpy})_{3}\right]^{2+}, \mathrm{Fe}(1,10-\text { phen })_{3}\right]^{2+}$.

Similar shifting of these MLCT bands and the brown color in 1- $\wedge-(\mathrm{S}, \mathrm{S}, \mathrm{S}, \mathrm{S})-\mathrm{Co}^{2+} / \mathrm{1}-\Delta-(\mathrm{R}, \mathrm{R}, \mathrm{R}, \mathrm{R})-\mathrm{Co}^{2+}$ and $2-\wedge-(\mathrm{S}, \mathrm{S}, \mathrm{S}, \mathrm{S})-\mathrm{Co}^{2+}$ / 2- $\Delta-(\mathrm{R}, \mathrm{R}, \mathrm{R}, \mathrm{R})-\mathrm{Co}^{2+}$ complexes are observed and are due to the strong field behavior of bis(arylethylimino)pyridine ligands, 1 and 2, in a similar manner to other $\mathrm{Co}^{2+}$-complexes with related ligands: $\left[\mathrm{Co}(\mathrm{bpy})_{3}\right]^{2+}$ and $\left[\mathrm{Co}(1,10-\text { phen })_{3}\right]^{2+}$. For all $\mathrm{Co}^{2+}$ complexes, the spin-allowed transitions are obscured by MLCT bands.

Circular Dichroism -CD- spectra The circular dichroism (CD) spectra measured in acetonitrile confirm the optical activity and enantiomeric nature of 1- $\wedge-(S, S, S, S)-M^{2+} / \mathbf{1}-\Delta-(R, R, R, R)-M^{2+}$ and $2-\wedge-(S, S, S, S)-M^{2+} / 2-\Delta-(R, R, R, R)-M^{2+}$ chiral complexes (Figure 1) :

- in acetonitrile solution, the complexes retain their chirality as the enantiomeric pairs present the same absorption maxima with opposite signs. Their spectra show negative or positive Cotton effects depending on the sense of chirality;

- the absorption bands in the spectra of the 2- $\wedge-(\mathrm{S}, \mathrm{S}, \mathrm{S}, \mathrm{S})-\mathrm{M}^{2+} /$ 2- $\Delta-(R, R, R, R)-M^{2+}$ complexes are weaker in intensity compared to those in the spectra of 1- $\Lambda-(S, S, S, S)-M^{2+} / 1-\Delta-(R, R, R, R)-M^{2+}$. This fact may be correlated with the better $\pi$-acceptor character of the ligand $\mathbf{1}$ and, consequently, with the ability to exert a stronger field in its complexes. In a series of ligands with aromatic constituents, the antibonding orbital (LUMO, $\pi^{*}$ ) is at lower energy values as the conjugate system is more extended, therefore the LUMO orbitals are energetically closer to the metal orbital $t_{2 g}$ and the overlapping $\pi$ metal-ligand is strong. The result of this stronger interaction with the $\pi$-acceptor ligand is an increase in transition energy.

- the absorption bands with maxima situated around $485 \mathrm{~nm}$ are present both in DC and UV-Vis spectra of $\mathrm{Fe}^{2+}$-complexes (Fig.1c,d). These absorption bands were attributed to the spinallowed transition ${ }^{1} \mathrm{~A}_{1 \mathrm{~g}} \rightarrow{ }^{1} \mathrm{~T}_{1 \mathrm{~g}}$ for $\mathrm{Fe}^{2+}, d^{6}$, LS.
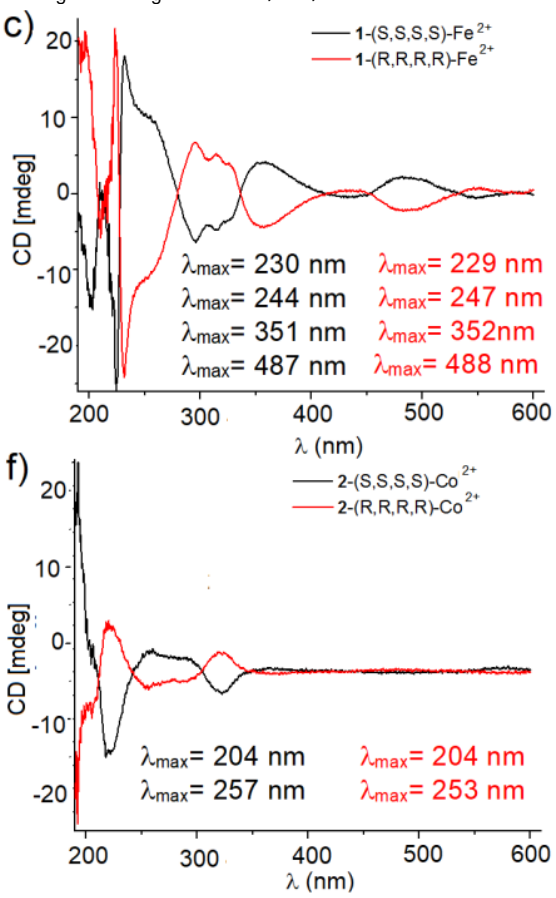

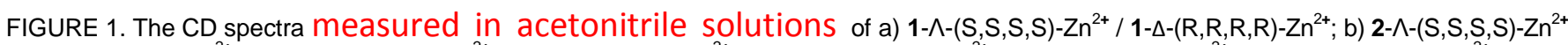

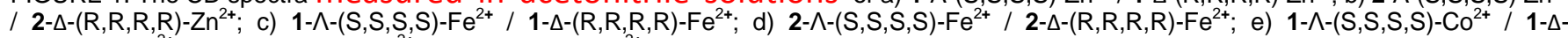
$\left.(\mathrm{R}, \mathrm{R}, \mathrm{R}, \mathrm{R})-\mathrm{Co}^{2+} ; \mathrm{f}\right) \mathrm{2-}-\mathrm{-}-(\mathrm{S}, \mathrm{S}, \mathrm{S}, \mathrm{S})-\mathrm{Co}^{2+} / \mathbf{2 - \Delta}-(\mathrm{R}, \mathrm{R}, \mathrm{R}, \mathrm{R})-\mathrm{Co}^{2+}$ complexes. 
TABLE $2 d-d$ spin-allowed transitions and MLCT bands for 1- $-(\mathrm{S}, \mathrm{S}, \mathrm{S}, \mathrm{S})-\mathrm{M}^{2+} / \mathbf{1}-\Delta-(\mathrm{R}, \mathrm{R}, \mathrm{R}, \mathrm{R})-\mathrm{M}^{2+}$ and $2-\Lambda-(\mathrm{S}, \mathrm{S}, \mathrm{S}, \mathrm{S})-\mathrm{M}^{2+} / \mathbf{2}-\Delta-(\mathrm{R}, \mathrm{R}, \mathrm{R}, \mathrm{R})-\mathrm{M}^{2+}, \mathrm{M}=\mathrm{Co} \mathrm{C}^{2+}, \mathrm{Fe} \mathrm{e}^{2+}$

\begin{tabular}{|c|c|c|c|c|c|c|c|}
\hline Complex & $\begin{array}{l}\text { 1- } \Delta-(R, R, R, R)- \\
\mathrm{Fe}^{2+}\end{array}$ & $\begin{array}{l}\text { 1-^-(S,S,S,S)- } \\
\mathrm{Fe}^{2+}\end{array}$ & $\begin{array}{l}\text { 2- } \Delta-(R, R, R, R)- \\
\mathrm{Fe}^{2+}\end{array}$ & $\begin{array}{l}2-\wedge-(\mathrm{S}, \mathrm{S}, \mathrm{S}, \mathrm{S})- \\
\mathrm{Fe}^{2+}\end{array}$ & $\begin{array}{l}\text { 1- } \Delta-(\mathrm{R}, \mathrm{R}, \mathrm{R}, \mathrm{R})-\mathrm{Co}^{2+} \\
1-\Lambda-(\mathrm{S}, \mathrm{S}, \mathrm{S}, \mathrm{S})-\mathrm{Co}^{2+}\end{array}$ & $\begin{array}{l}2-\Delta-(R, R, R, R)- \\
\mathrm{Co}^{2+}\end{array}$ & $2-\Lambda-(\mathrm{S}, \mathrm{S}, \mathrm{S}, \mathrm{S})-\mathrm{Co}^{2+}$ \\
\hline \multirow[t]{4}{*}{$\begin{array}{l}\text { Transitions } \\
\text { (kK) }\end{array}$} & $\begin{array}{c}35.57(281 \mathrm{~nm}) \\
{ }^{1} \mathrm{~A}_{1 \mathrm{~g}} \rightarrow{ }^{1} \mathrm{~T}_{2 \mathrm{~g}} \\
\left(\mathrm{MLCT} \mathrm{d}-\pi^{\star}\right) \\
\end{array}$ & $\begin{array}{c}35.57(281 \mathrm{~nm}) \\
{ }^{1} \mathrm{~A}_{1 \mathrm{~g}} \rightarrow{ }^{1} \mathrm{~T}_{2 \mathrm{~g}} \\
\left(\mathrm{MLCT} \mathrm{d}-\pi^{\star}\right) \\
\end{array}$ & $\begin{array}{c}30.76(325 \mathrm{~nm}) \\
{ }^{1} \mathrm{~A}_{1 \mathrm{~g}} \rightarrow{ }^{1} \mathrm{~T}_{2 \mathrm{~g}} \\
\left(\mathrm{MLCT} \mathrm{d}-\pi^{\star}\right) \\
\end{array}$ & $\begin{array}{c}30.76(325 \mathrm{~nm}) \\
{ }^{1} \mathrm{~A}_{1 \mathrm{~g}} \rightarrow{ }^{1} \mathrm{~T}_{2 \mathrm{~g}} \\
\left(\mathrm{MLCT} \mathrm{d}-\pi^{\star}\right) \\
\end{array}$ & $\begin{array}{l}35.57(281 \mathrm{~nm}) \\
\text { MLCT d- } \pi^{*}\end{array}$ & $\begin{array}{l}33.32(300 \mathrm{~nm}) \\
\text { MLCT d- } \pi^{\star}\end{array}$ & $\begin{array}{l}33.06(303 \mathrm{~nm}) \\
\text { MLCT d- } \pi^{\star}\end{array}$ \\
\hline & $\begin{array}{c}20.67(484 \mathrm{~nm}) \\
{ }^{1} \mathrm{~A}_{1 \mathrm{~g}} \rightarrow{ }^{1} \mathrm{~T}_{1 \mathrm{~g}} \\
\end{array}$ & $\begin{array}{c}20.67(484 \mathrm{~nm}) \\
{ }^{1} \mathrm{~A}_{1 \mathrm{~g}} \rightarrow{ }^{1} \mathrm{~T}_{1 \mathrm{~g}} \\
\end{array}$ & $\begin{array}{c}20.83(480 \mathrm{~nm}) \\
{ }^{1} \mathrm{~A}_{1 \mathrm{~g}} \rightarrow{ }^{1} \mathrm{~T}_{1 \mathrm{~g}} \\
\end{array}$ & $\begin{array}{c}20.83(480 \mathrm{~nm}) \\
{ }^{1} \mathrm{~A}_{1 \mathrm{~g}} \rightarrow{ }^{1} \mathrm{~T}_{1 \mathrm{~g}} \\
\end{array}$ & & & \\
\hline & $\begin{aligned} & 16.45(608 \mathrm{~nm}) \\
&{ }^{1} \mathrm{~A}_{19} \rightarrow{ }^{3} \mathrm{~T}_{1 \mathrm{~g}} \\
&\end{aligned}$ & $\begin{array}{c}16.45(608 \mathrm{~nm}) \\
{ }^{1} \mathrm{~A}_{1 \mathrm{~g}} \rightarrow{ }^{3} \mathrm{~T}_{1 \mathrm{~g}} \\
\end{array}$ & $\begin{array}{c}16.58(603 \mathrm{~nm}) \\
{ }^{1} \mathrm{~A}_{1 \mathrm{~g}} \rightarrow{ }^{3} \mathrm{~T}_{1 \mathrm{~g}} \\
\end{array}$ & $\begin{array}{c}16.58(603 \mathrm{~nm}) \\
{ }^{1} \mathrm{~A}_{1 \mathrm{~g}} \rightarrow{ }^{3} \mathrm{~T}_{19}\end{array}$ & & & \\
\hline & $\begin{array}{l}14.2(705 \mathrm{~nm}) \\
{ }^{1} \mathrm{~A}_{1 \mathrm{~g}} \rightarrow{ }^{3} \mathrm{~T}_{2 g} \\
\end{array}$ & & $\begin{array}{c}14.09(710 \mathrm{~nm}) \\
{ }^{1} \mathrm{~A}_{1 \mathrm{~g}} \rightarrow{ }^{3} \mathrm{~T}_{2 \mathrm{~g}} \\
\end{array}$ & $\begin{array}{c}14.2(705 \mathrm{~nm}) \\
{ }^{1} \mathrm{~A}_{1 \mathrm{~g}} \rightarrow{ }^{3} \mathrm{~T}_{2 g} \\
\end{array}$ & & & \\
\hline
\end{tabular}

a)
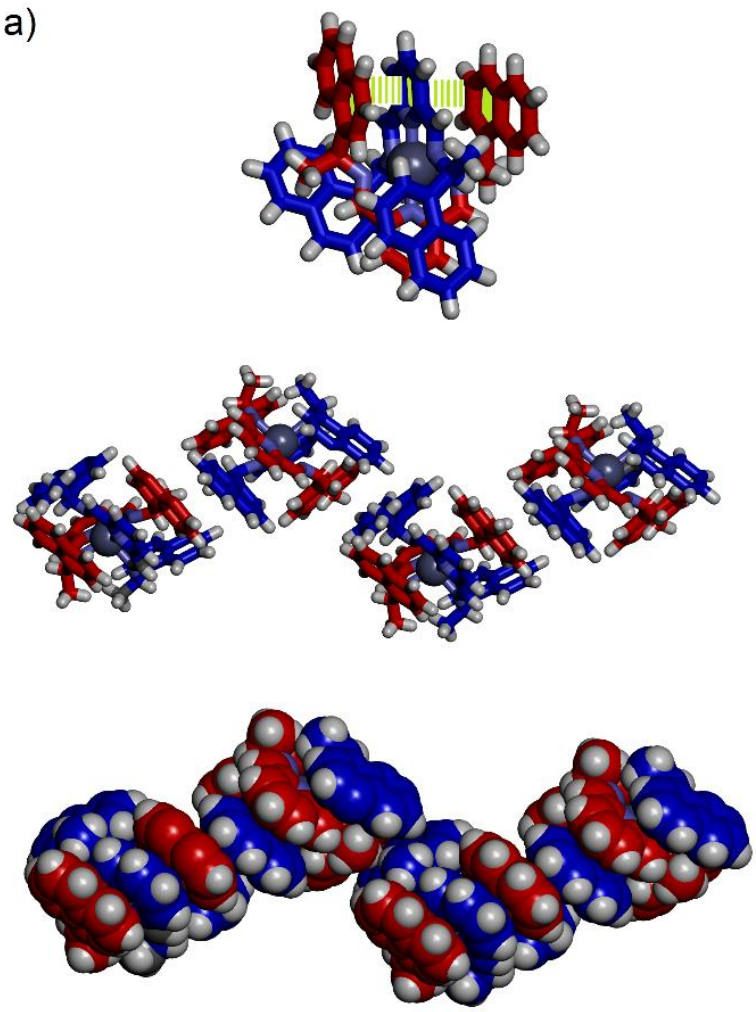

b)
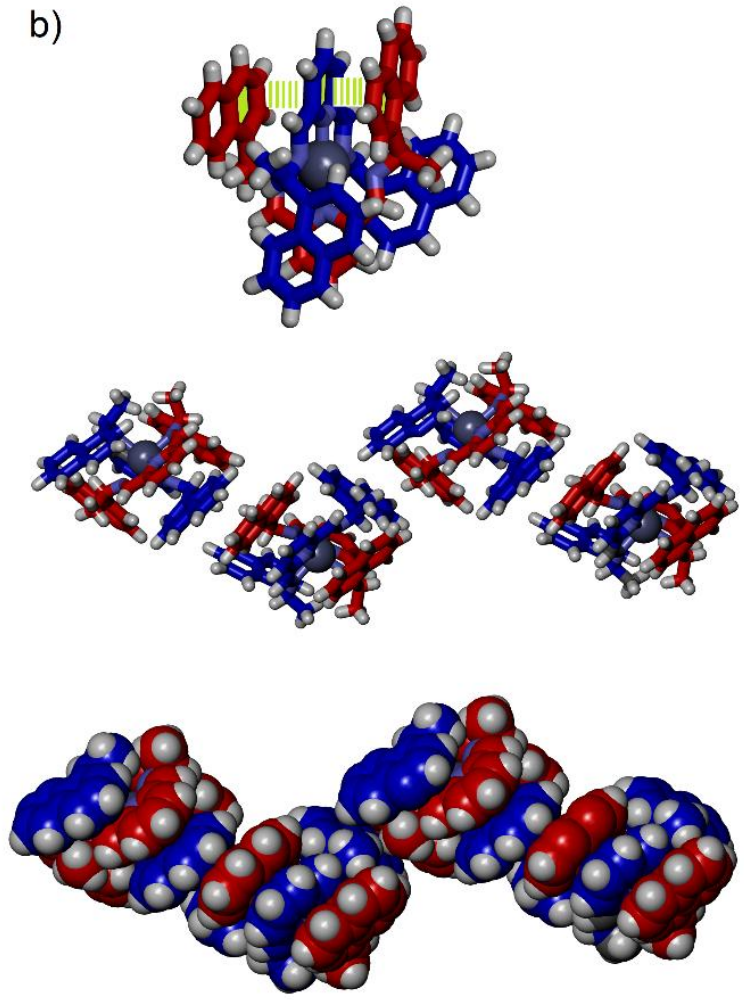

FIGURE 2 Homochiral duplexes (top) and side view in stick (middle) and CPK (bottom) representation of the crystal packing of a) 1-^-(S,S,S,S)$\mathrm{Zn}^{2+}$ and b) 1- $\Delta-(\mathrm{R}, \mathrm{R}, \mathrm{R}, \mathrm{R}) \mathrm{Zn}^{2+}$ complexes. Green lines represent $\pi-\pi$ interactions.

- for the $\mathrm{Zn}^{2+}$-complexes (Fig.1a,b), in the absence of Crystal Field Stabilisation Energy CFSE $\left(d^{10}\right)$, the absorption bands are attributed to the allowed electronic transitions ${ }^{1} \mathrm{~L}_{a}$ and ${ }^{1} \mathrm{~B}_{\mathrm{b}}$ from the chromophore moieties of the chiral ligands 1 and $2:{ }^{1} \mathrm{~L}_{\mathrm{a}}=200$ $\mathrm{nm}$ and $206 \mathrm{~nm},{ }^{1} \mathrm{~B}_{\mathrm{b}}=267-268 \mathrm{~nm}$ for phenyl chromophore, ${ }^{1} \mathrm{~L}_{\mathrm{a}}=234-235 \mathrm{~nm},{ }^{1} \mathrm{~B}_{\mathrm{b}}=348$ and $336 \mathrm{~nm}$ for naphthyl chromophore. ${ }^{57}$ Compared to the CD spectra of the constituent chromophores of the ligands, the chiral amines: R-(+)-1-/S-(-) -1-phenylethylamine and $\mathrm{R}-(+)-1-/ \mathrm{S}-(-)-1$-naphthylethylamine), these maxima are red-shifted ( ${ }^{1} B_{b}=269 \mathrm{~nm}, 261 \mathrm{~nm}$-phenyl, ${ }^{1} \mathrm{~B}_{\mathrm{b}}=336 \mathrm{~nm}, 282 \mathrm{~nm}$-naphthyl), probably due to the electronic interactions (charge transfer or $\pi-\pi$ stacking) chromophorepyridine.$^{58}$ The same behavior also occurs in the CD spectra of $\mathrm{Fe}^{2+}$ (Fig.1c,d) and $\mathrm{Co}^{2+}$ (Fig.1e,f) complexes.

X-ray Single Crystal Diffraction Structures. The crystal structures of the complexes 1- $\Lambda-(S, S, S, S)-M^{2+} / 1-\Delta-(R, R, R, R)-$ $\mathrm{M}^{2+} \quad \mathrm{M}^{2+}=\mathrm{Co}^{2+}, \quad \mathrm{Fe}^{2+}, \quad \mathrm{Zn}^{2+}, \quad 2-\Lambda-(\mathrm{S}, \mathrm{S}, \mathrm{S}, \mathrm{S})-\mathrm{Fe}^{2+}$ and 2- $\Delta-$ $(\mathrm{R}, \mathrm{R}, \mathrm{R}, \mathrm{R})-\mathrm{Co}^{2+}$ were determined from crystals obtained from the acetonitrile/i-propylether solutions at room temperature. Labelled asymmetric units, selected bond lengths and angles are described in Tables S1-S4 (Supporting Information). The molecular and the crystal packing structures are presented in Figures 2-5.

$1-\wedge-(S, S, S, S)-Z^{2+}$ and $1-\Delta-(R, R, R, R)-Z^{2+} \quad$ complexes belong to the orthorhombic non-centrosymmetric space group $P 22_{1} 2_{1}(\# 18)$, and the crystallographic data are listed in Table S1. The asymmetric unit consists in both cases of $[1-\wedge-(S, S, S, S)-$ $\mathrm{Zn}]^{2+}$ or $[1-\Delta-(\mathrm{R}, \mathrm{R}, \mathrm{R}, \mathrm{R})-\mathrm{Zn}]^{2+}$ cations and two $\mathrm{CF}_{3} \mathrm{SO}_{3}^{-}$anions. The $\mathrm{Zn}^{2+}-\mathrm{N}_{\text {Pyridine }}$ and $\mathrm{Zn}^{2+}-\mathrm{N}_{\text {imine }}$ distances vary between 2.002(4)-2.013(4) $\AA$ and 2.2587(17)-2.4055(17) $\AA$, respectively.

$1-\Lambda-(S, S, S, S)-F^{2+}$ and 1- $\Delta-(R, R, R, R)-\mathrm{Fe}^{2+}$ complexes crystallize in the orthorhombic non-centrosymmetric space group $P 2{ }_{1} 2_{1} 2_{1}$ (\#19) (Table S2). The asymmetric unit consists in both cases of $[1-\Lambda-(S, S, S, S)-F e]^{2+}$ or $[1-\Delta-(R, R, R, R)-F e]^{2+}$ cations, two $\mathrm{BF}_{4}$ - anions, and two acetonitrile molecules. The geometric parameters imply that $1-\wedge-(S, S, S, S)-F^{2+}$ and $1-\Delta-(R, R, R, R)-$ $\mathrm{Fe}^{2+}$ complexes are low-spin at $175 \mathrm{~K}$ and the $\mathrm{Fe}-\mathrm{N}$ distances are of typical values for LS complexes of $\mathrm{Fe}^{2+}$ with pyridine-type ligands. The average $\mathrm{Fe}^{2+}-\mathrm{N}_{\text {Pyridine }}$ and $\mathrm{Fe}^{2+}-\mathrm{N}_{\text {imine }}$ distances are $1.8519(17)-1.880(2) \quad \AA \quad$ and $1.9457(17)-2.1073(18) \quad \AA$, respectively. 
1- $\wedge-(\mathrm{S}, \mathrm{S}, \mathrm{S}, \mathrm{S})-\mathrm{Co}^{2+}$ and 1- $\Delta-(\mathrm{R}, \mathrm{R}, \mathrm{R}, \mathrm{R})-\mathrm{Co}^{2+}$ crystals belong to the enantiomorphic space group pair $P 6_{1} 22 / P 6_{5} 22$. The asymmetric unit consists in both cases of $[1-\Lambda-(S, S, S, S)-C o]^{2+}$ and $[1-\Delta-(R, R, R, R)-C o]^{2+}$ cations and two $B_{4}-$ anions (Table $\left.S 3\right)$. The Co-N distances, $\mathrm{Co}^{2+}-\mathrm{N}_{\text {Pyridine }}=1.848(3)-1.925(5) \AA$ and $\mathrm{Co}^{2+}-\mathrm{N}_{\text {imine }}=2.017(3)-2.318(4) \AA$, indicate that $1-\Lambda-(\mathrm{S}, \mathrm{S}, \mathrm{S}, \mathrm{S})-$ $\mathrm{Co}^{2+}$ and 1- $\Delta-(\mathrm{R}, \mathrm{R}, \mathrm{R}, \mathrm{R})-\mathrm{Co}^{2+}$ are, as expected, low-spin complexes at $175 \mathrm{~K}$.

2- $\wedge-(\mathrm{S}, \mathrm{S}, \mathrm{S}, \mathrm{S})-\mathrm{Fe}^{2+}$ and 2- $\Delta-(\mathrm{R}, \mathrm{R}, \mathrm{R}, \mathrm{R})-\mathrm{Co}^{2+}$ crystallize in the orthorhombic non-centrosymmetric space group $P 2{ }_{1} 2_{1} 2_{1}$ (\#19) (Table S4). Each $\mathrm{Fe}^{2+} / \mathrm{Co}^{2+}$ ion binds two bis(phenylethylimine)pyridine ligands, 2, and has a pseudooctahedral coordination geometry, but the complexes did not crystallize similarly: both complexes are solvates but 2- $\Delta$ $(\mathrm{R}, \mathrm{R}, \mathrm{R}, \mathrm{R})-\mathrm{Co}^{2+}$ crystallizes with only one acetonitrile solvent molecule, whereas two acetonitrile molecules are present in the structure of $2-\wedge-(S, S, S, S)-F^{2+}$

In all duplex structures the $\mathrm{M}^{2+}$ metal ions are fully coordinated by two ligands arranged into orthogonal planes and present an octahedral coordination geometry. Each ligand in the all-cis configuration serves as a tridentate ligand and coordinates meridionally to the metal ion with one pyridine, and two imine nitrogen atoms. Continuous shape measurements analysis ${ }^{59,60}$ showed that all transition metal ions, $\mathrm{M}^{2+}=\mathrm{Zn}^{2+}, \mathrm{Fe}^{2+}, \mathrm{Co}^{2+}$ display distorted octahedral coordination environments (Table S5, Figs S3-S4), and among these, $\mathrm{Fe}^{2+}$-complexes (LS, $d^{6}$ ) are the less distorted from ideal octahedron, whereas $\mathrm{Co}^{2+}\left(\mathrm{LS}, d^{7}\right)$ with one electron in the antibonding $e_{g}$ orbitals and $\mathrm{Zn}^{2+}\left(d^{19}\right)$ present greater degrees of distortion.

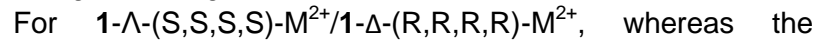
average $\mathrm{Fe}^{2+}-\mathrm{N}_{\text {Pyridine }}$ and $\mathrm{Co}^{2+}-\mathrm{N}_{\text {Pyridine }}$ distances are similar:

a)
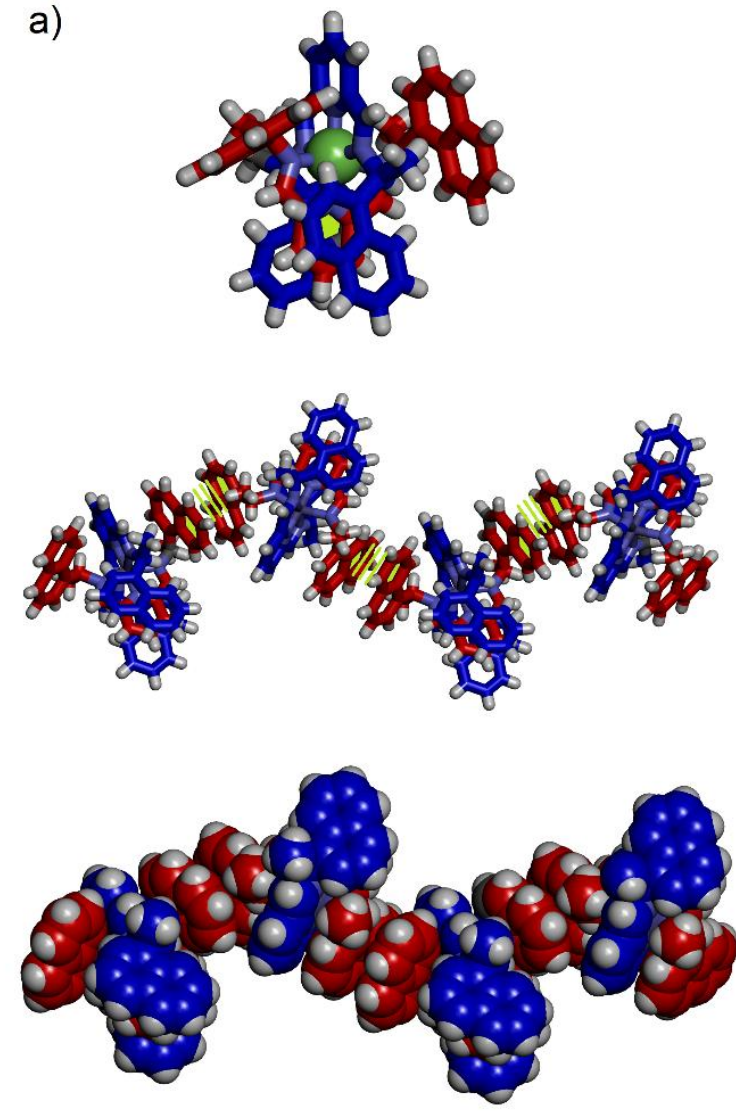

1.852/1.848 $\AA$, the average $\mathrm{Zn}^{2+}-\mathrm{N}_{\text {Pyridine }}$ distance is much longer: $2.002 \AA$. The $\mathrm{M}^{2+}-\mathrm{N}_{\text {imine }}$ distances are progressively increasing as following: $\mathrm{Fe}^{2+}-\mathrm{N}_{\text {imine }}<\mathrm{Co}^{2+}-\mathrm{N}_{\text {imine }}<\mathrm{Zn}^{2+}-\mathrm{N}_{\text {imine }}$ distances of 1.946 $\AA$, $2.017 \AA$ and $2.258 \AA$, respectively. These fairly different geometrical parameters and the $\mathrm{M}^{2+}$ coordination behaviour (i.e. distorted symmetry for $\mathrm{Fe}^{2+} / \mathrm{Co}^{2+}$ and the lack of CFSE for $\mathrm{Zn}^{2+}$ ) lead to slight differences in the spatial disposition of lateral naphthyl arms. As an important consequence the double helix complexes are not isostructural: they crystallize in different space groups ( $P 22_{1} 2_{1}, P 2_{1} 2_{1} 2_{1}, P 6_{1} 22 / P 6_{5} 22$ respectively). This fact is more evident for the crystals of enantiomeric pairs 1- $\Lambda$ -

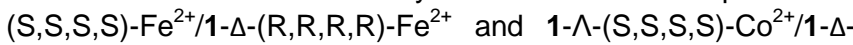
$(R, R, R, R)-\mathrm{Co}^{2+}$ that are not isostructural, although they have been synthesized under identical conditions: same solvent system $\left(\mathrm{CD}_{3} \mathrm{CN} / i\right.$-propylether) and same counterion $\left(\mathrm{BF}_{4}^{-}\right)$.

The methyl- $\mathrm{CH}^{*}$ chiral spacer in the structure of the ligands is important for holding and stabilizing the duplex formation by the internal $\pi-\pi$ stacking. As a general rule, in the crystal the two ligands are strongly intertwined stabilizing the duplex superstructures by internal $\pi-\pi$ stacking interactions. The relative position of the duplex ligands allows a partial (1-^-(S,S,S,S)-Fe ${ }^{2+}$ 1- $\Delta-(\mathrm{R}, \mathrm{R}, \mathrm{R}, \mathrm{R}) \mathrm{Fe}^{2+}$ 2- $\Lambda-(\mathrm{S}, \mathrm{S}, \mathrm{S}, \mathrm{S})-\mathrm{Fe}^{2+}$ and $\left.2-\Delta-(\mathrm{R}, \mathrm{R}, \mathrm{R}, \mathrm{R})-\mathrm{Co}^{2+}\right)$ or a total (1-^-(S,S,S,S)-Zn ${ }^{2+}, 1-\Delta-(R, R, R, R)-Z^{2+}, 1-\Lambda-(S, S, S, S)-$ $\mathrm{Co}^{2+}$ and $\left.1-\Delta-(R, R, R, R)-C^{2+}\right)$ internal overlap between the naphthyl or phenyl moieties and the central pyridine moiety of a vicinal ligand via $\pi-\pi$ stacking aromatic interactions with an average centroid-centroid distances of 3.4-3.7 $\AA$, corresponding to van der Waals contacts: face-to-face $\pi-\pi$, heteroaromatic (py)-aromatic interactions and $\mathrm{CH} \cdots \pi$ (y-interaction) (Fig. S5S8). ${ }^{61}$

b)
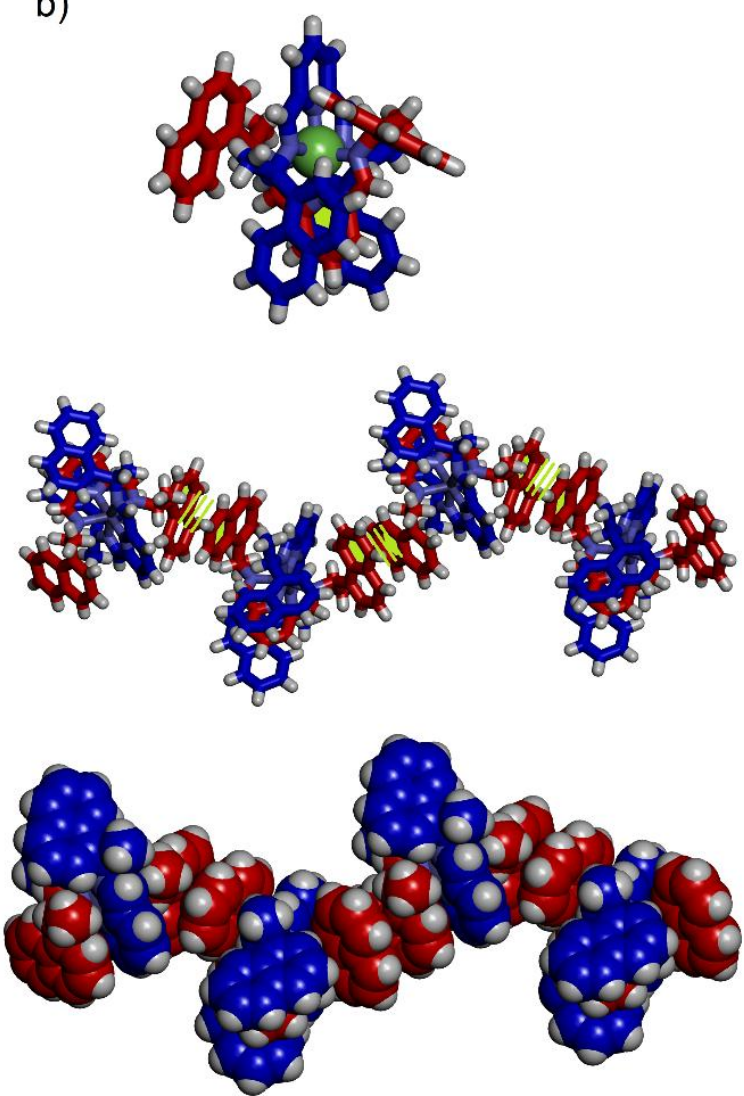

FIGURE 3 Homochiral duplexes (top) and side view in stick (middle) and CPK (bottom) representation of the crystal packing of a) 1-^-(S,S,S,S)$\mathrm{Fe}^{2+}$ and $\left.b\right) 1-\Delta-(R, R, R, R)-\mathrm{Fe}^{2+}$ complexes. Green lines represent $\Pi-\pi$ interactions. 
Chirality

a)

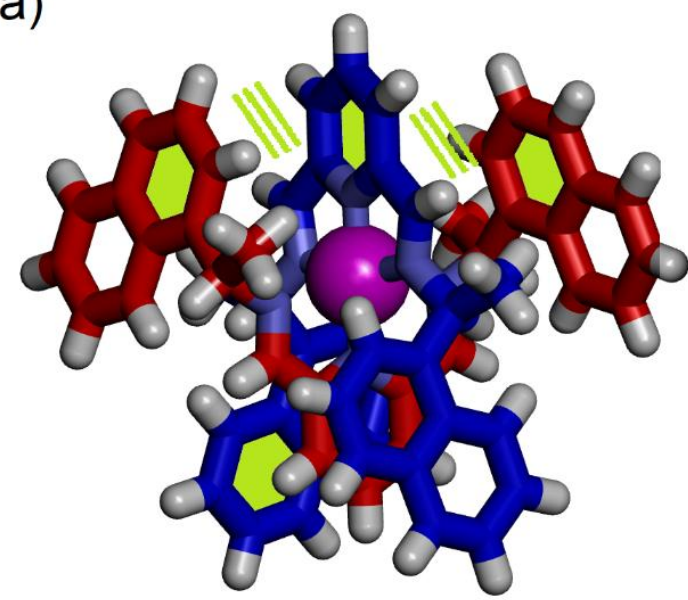

b)

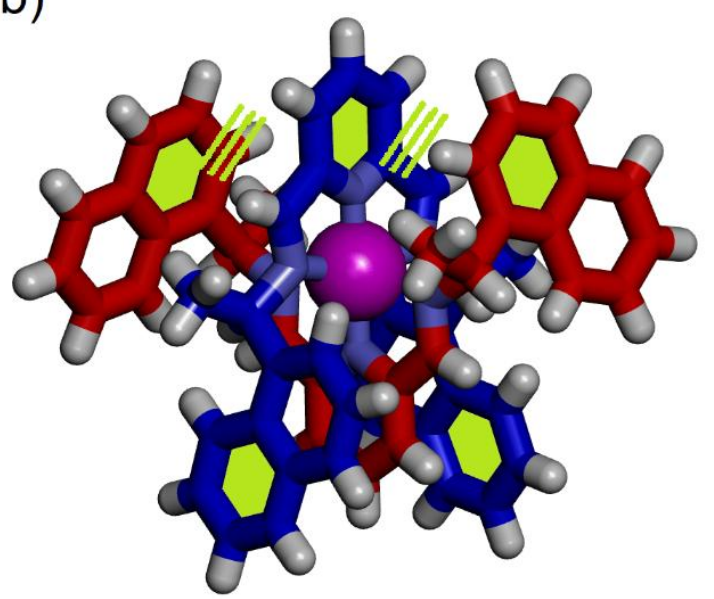

c)
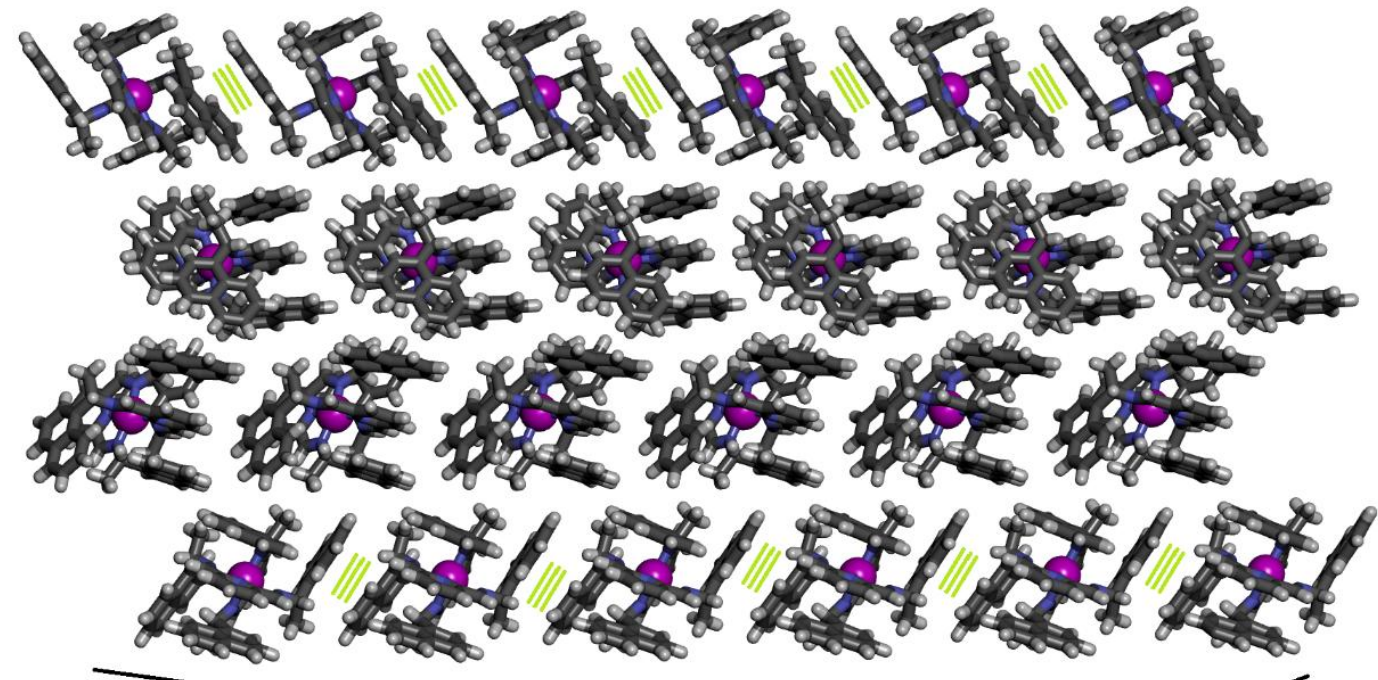

d)

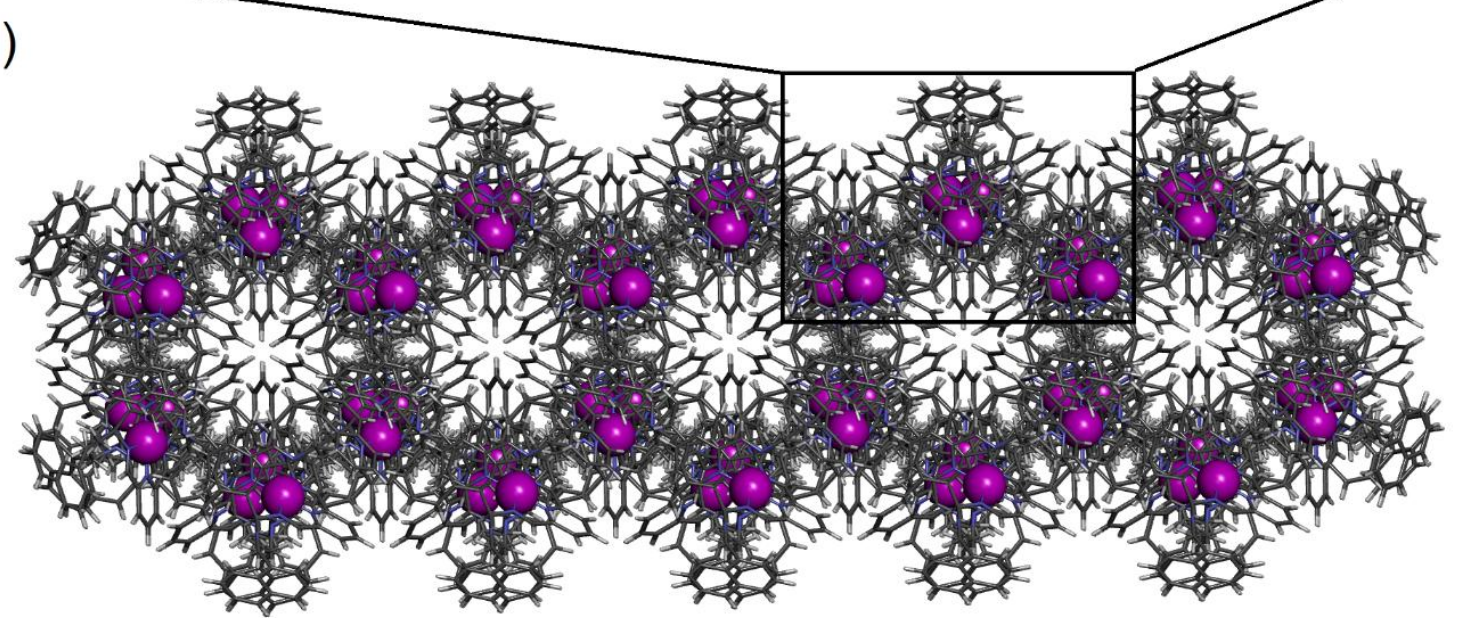

FIGURE 4 Homochiral duplexes a) 1- $\Lambda-(S, S, S, S)-C o^{2+}$ and b) $1-\Delta-(R, R, R, R)-C o^{2+}$; c) side view and d) top view in stick representation of the crystal packing of complexes. Green lines represent $\pi-\pi$ interactions.

Between peripheral aromatic rings (naphthyl for 1- $\Lambda$ $(\mathrm{S}, \mathrm{S}, \mathrm{S}, \mathrm{S})-\mathrm{M}^{2+} / \mathbf{1}-\Delta-(\mathrm{R}, \mathrm{R}, \mathrm{R}, \mathrm{R})-\mathrm{M}^{2+}$ or phenyl for $2-\Lambda-(\mathrm{S}, \mathrm{S}, \mathrm{S}, \mathrm{S})-\mathrm{Fe}^{2+}$ and $\left.2-\Delta-(R, R, R, R)-C^{2+}\right)$ intermolecular associations through $\mathrm{CH} \cdots \pi$ (T-interaction) with average centroid-edge distances of 3.4-3.7 ̊̊ are established (Fig. S5-S8). ${ }^{61}$

In the case of partial internal overlap, the external $\pi-\pi$ stacking interactions are occurring between communicating duplex structures. It is resulting in the formation of left handed 1$\Lambda-(\mathrm{S}, \mathrm{S}, \mathrm{S}, \mathrm{S})-\mathrm{Fe}^{2+}$ (Fig. 3), 2- $\left.\Lambda-(\mathrm{S}, \mathrm{S}, \mathrm{S}, \mathrm{S})-\mathrm{Fe}^{2+}\right)$ (Fig. 5) or right handed 1- $\Delta-(\mathrm{R}, \mathrm{R}, \mathrm{R}, \mathrm{R})-\mathrm{Fe}^{2+}$ (Fig. 3), and $2-\Delta-(\mathrm{R}, \mathrm{R}, \mathrm{R}, \mathrm{R})-\mathrm{Co}^{2+}$ (Fig.
5), single helix superstructures that are present in the crystal structure.

In a different manner, in the crystals of 1- $\Lambda-(\mathrm{S}, \mathrm{S}, \mathrm{S}, \mathrm{S})-\mathrm{Zn}^{2+}, \mathbf{1 -}$ $\Delta-(\mathrm{R}, \mathrm{R}, \mathrm{R}, \mathrm{R})-\mathrm{Zn}^{2+}, \quad 1-\Lambda-(\mathrm{S}, \mathrm{S}, \mathrm{S}, \mathrm{S})-\mathrm{Co}^{2+}$ and 1- $\Delta-(\mathrm{R}, \mathrm{R}, \mathrm{R}, \mathrm{R}) \mathrm{Co}^{2+}$ complexes, the communication between duplexes which are mostly internally stacked is disrupted; each duplex being closely packed with two neighbouring ones by weak van der Waals contacts while the external $\pi-\pi$ stacking aromatic interactions are completely suppressed in the frameworks. (Figs. 2,4). 
a)
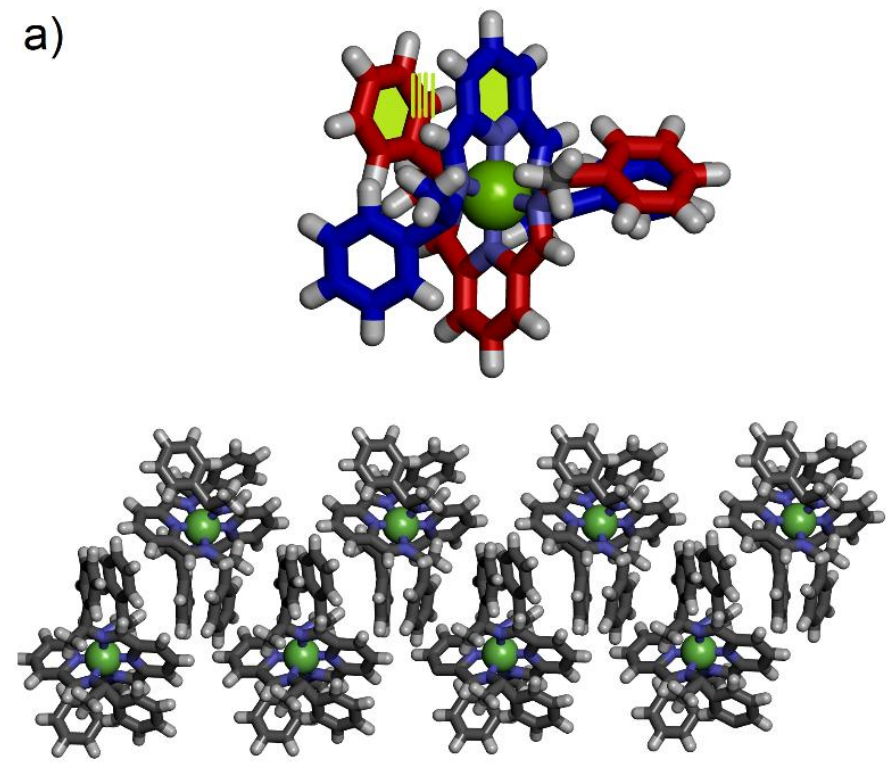

FIGURE 5 Homochiral duplexes (top) and side view of the crystal packing (bottom) of a) $2-\wedge-(S, S, S, S)-F^{2+}$ and b) $2-\Delta-(R, R, R, R)-C^{2+}$ complexes.

\section{Conclusion}

In conclusion we have demonstrated in this paper that the molecular chirality is transferred to chiral duplex superstructures that can be generated in solution and solid state single-crystals via a combination of metal-ion coordination and weak $\pi-\pi$ stacking and van der Waals interactions. Hierarchical supramolecular organization is promoted by the formation of metallosupramolecular duplexes, stabilized by internal $\pi-\pi$ stacking. The introduction of a chiral spacer between imine moiety and aryl groups, induce a spatial orientation of the lateral aromatic arms that is clearly important for internal holding and stabilization of the duplex formation by $\pi-\pi$ stacking. When such internal interactions are dominant, the external $\pi-\pi$ stacking communication is completely removed and non-communicating duplex structures are present in the crystal. Long-range 3D supramolecular structure propagation is favoured when both partial internal overlapping and external $\pi-\pi$ stacking are present, leading to the formation of robust single-helical configurations or tubular packed architectures. The use of multiple supramolecular interactions provides a very powerful platform for the transfer of chiral information from molecular to supramolecular level. The internal robustness of the duplexes is mainly responsible for the transmission of the supramolecular homochiral order and is reminiscent with sliding biological processes along homochiral 3D hypersurfaces occur in the formation of chiral biological relevant species at the nanolevel. ${ }^{62}$

Acknowledgements

This work was supported by Agence Nationale de la Recherche ANR-15-CE29-0009 DYNAFUN.

\section{Supporting information}

b)
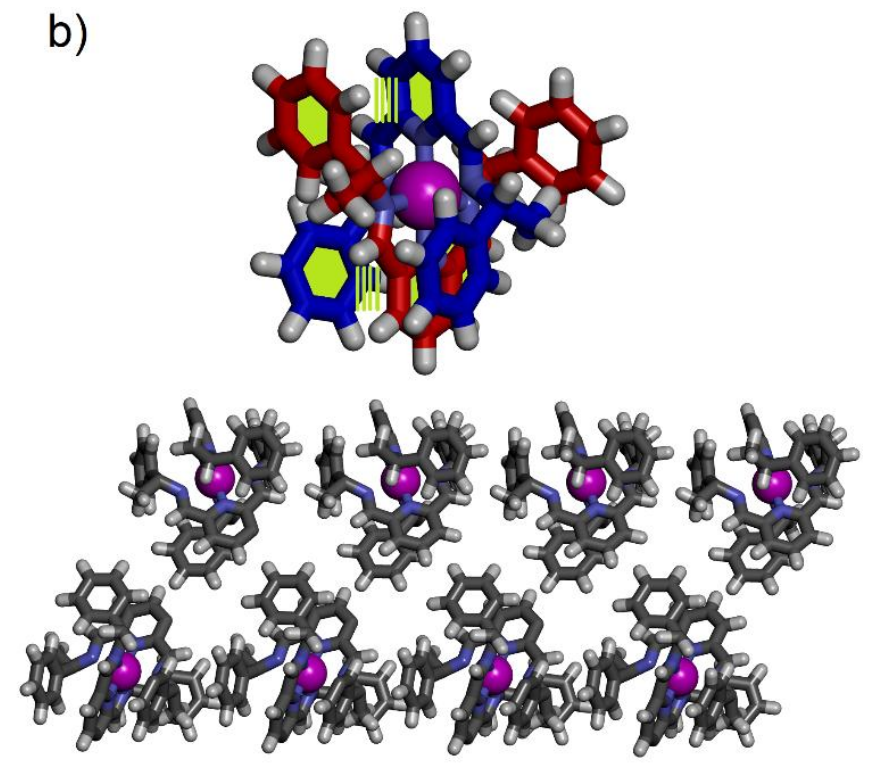

Additional supporting information may be found in the online version of this article at the publisher's website.

\section{REFERENCES AND NOTES}

1. Amouri H, Gruselle M. Chirality in Transition Metal Chemistry: Molecules, Supramolecular Assemblies and Materials. New York: John Wiley \& Sons; 2008.

2. Zhang X, Yin J, Yoon J. Recent Advances in Development of Chiral Fluorescent and Colorimetric Sensors. Chem Rev 2014;114:4918-4959.

3. a) Dragna JM, Pescitelli G, Tran L, Lynch VM, Anslyn EV, Di Bari L. In Situ Assembly of Octahedral $\mathrm{Fe}^{2+}$ Complexes for the Enantiomeric Excess Determination of Chiral Amines Using Circular Dichroism Spectroscopy. J Am Chem Soc 2012;134:4398-4407. b) Nieto S, Dragna JM, Anslyn EV. A Facile CD Protocol for Rapid Determination of Enantiomeric Excess and Concentration of Chiral Primary Amines. Chem Eur J 2010:16(1):227-232. c) Dragna JM, Gade AM, Tran L, Lynch VM, Anslyn EV. Chiral Amine Enantiomeric Excess Determination Using Self-Assembled Octahedral $\mathrm{Fe}^{2+-}$ Imine Complexes. Chirality 2015;27:294-298.

4. Chen X, Lu Z. Recent advances in chiral imino-containing ligands for metal-catalyzed asymmetric transformations. Org Biomol Chem 2017;15:2280-2306.

5. Cruchter T, Larionov VA. Asymmetric catalysis with octahedral stereogenic-at-metal complexes featuring chiral ligands. Coord Chem Rev 2018;376:95-113.

6. Crassous J. Chiral transfer in coordination complexes: towards molecular materials. Chem Soc Rev 2009;38:830845.

7. Aritake $\mathrm{Y}$, Nakayama T, Nishizuru H, Akitsu T. Observation of induced $C D$ on CdSe nanoparticles from chiral Schiff base $\mathrm{Ni}^{2+}, \mathrm{Cu}^{2+} \mathrm{Zn}^{2+}$ complexes. Inorg Chem Comm 2011;14:423425.

8. Aritake Y, Takanashi T, Yamazaki A, Akitsu T. Polarized spectroscopy and hybrid materials of chiral Schiff base $\mathrm{Ni}(2+), \mathrm{Cu}(2+), \mathrm{Zn}(2+)$ complexes with included or separated azo-groups. Polyhedron 2011;30:886-894.

9. Akitsu T, Itoh T. Polarized spectroscopy of hybrid materials of chiral Schiff base cobalt(2+), nickel(2+), copper(2+), and zinc(2+) complexes and photochromic azobenzenes in PMMA films. Polyhedron 2010;29:477-487.

10. Akitsu T. Photofunctional supramolecular solution systems of chiral Schiff base nickel(2+), copper(2+), and zinc(2+) complexes and photochromic azobenzenes. Polyhedron 2007;26:2527-2535. 
11. Onodera T, Akitsu T. Tuning of the optical properties of chiral Schiff base $\mathrm{Zn}(2+)$ complexes by substituents. Polyhedron 2013;59:107-114.

12. Albert J, Magali Cadena J, Granell JR, Solans X, Font-Bardia $M$. Optically active palladacycles containing imines derived from 1-(1-naphthyl)ethylamine: new resolving agents for $P$ chiral phosphines. Tetrahedron Asymmetry 2000;11:19431955.

13. Benito M, López C, Solans X, Font-Bardía M. Palladium(2+) compounds with planar chirality. X-Ray crystal structures of (+)- $(R)-\left[\left\{\left(\eta^{5}-\mathrm{C}_{5} \mathrm{H}_{4}\right)-\mathrm{CH}=\mathrm{N}-\mathrm{CH}(\mathrm{Me})-\mathrm{C}_{10} \mathrm{H}_{7}\right\} \mathrm{Fe}\left(\eta^{5}-\mathrm{C}_{5} \mathrm{H}_{5}\right)\right]$ and $(+)-\left(R_{p}, R\right)-\left[\mathrm{Pd}\left\{\left[(\mathrm{Et}-\mathrm{C}=\mathrm{C}-\mathrm{Et})_{2}\left(\eta^{5}-\mathrm{C}_{5} \mathrm{H}_{3}\right)-\mathrm{CH}=\mathrm{N}-\mathrm{CH}(\mathrm{Me})-\right.\right.\right.$ $\left.\left.\mathrm{C}_{10} \mathrm{H}_{7}\right] \mathrm{Fe}\left(\eta^{5}-\mathrm{C}_{5} \mathrm{H}_{5}\right)\right\} \mathrm{Cl}$. $\quad$ Tetrahedron Asymmetry 1998;9:4219-4238.

14. Bao L-Y, Hao S-J, Xi S-F, Yan X, Zhang H-X, Shen R, Gu ZG. Chiral supramolecular coordination cages as highperformance inhibitors against amyloid- $\beta$ aggregation. Chem Commun 2018;54:8725-8728.

15. Zhou X-Q, Li Y, Zhang D-Y, Nie Y, Li Z-J, Gu W, Liu X, Tian J-L, Yan S-P. Copper Complexes Based on Chiral Schiffbase Ligands: DNA/BSA Binding Ability, DNA Cleavage Activity, Cytotoxicity and Mechanism of Apoptosis, Eur J Med Chem 2016;114:244-256.

16. Anderson C, Crespo M, Rochon FD. Stereoselective oxidative addition of methyl iodide to chiral cyclometallated $\mathrm{Pt}^{2+}$ compounds derived from $(R)-(+)-1-(1-$ naphthyl ethylamine). Crystal structure of [PtMe\{3-(R)$\left.\left(\mathrm{C}_{10} \mathrm{H}_{7}\right) \mathrm{CHMeNCHC} \mathrm{H}_{2} \mathrm{~S}_{3} \mathrm{PPh}_{3}\right]$. J Organomet Chem 2001;631:164-174.

17. Khan NH, Pandya N, Prathap KJ, Kureshy RI, Abdi SHR, Mishra S, Bajaj HC. Chiral discrimination asserted by enantiomers of $\mathrm{Ni}(2+), \mathrm{Cu}(2+)$ and $\mathrm{Zn}(2+)$ Schiff base complexes in DNA binding, antioxidant and antibacterial activities. Spectrochim Acta A Mol Biomol Spectrosc 2011;81:199-208.

18. Muppidi VK, Zacharias PS, Pal S. Self-assembly of a pseudo-tetrahedral $\mathrm{Zn}(2+)$ complex with a chiral reduced Schiff base into a helical superstructure. Inorg Chem Commun 2005;8:543-547.

19. Strong ETJ, Price JT, Jones ND. Modular syntheses of chiral and achiral $\mathrm{C}, \mathrm{N}$-chelated $\mathrm{Pd}(2+)$-pyridinylidenes. Dalton Trans 2009;9123-9125.

20. Iglesias AL, Aguirre G, Somanathan R, Parra-Hake M. New chiral Schiff base-Cu(2+) complexes as cyclopropanation catalysts. Polyhedron 2004;23:3051-3062.

21. Becerra A, Contreras R, Carmona D, Lahoz FJ, GarciaOrduna P. Half-sandwich rhodium and iridium complexes containing homochiral imidazolyl-imine ligands: synthesis, characterization and catalytic applications. Dalton Trans 2013;42:11640-11651

22. Carmona D, Lahoz FJ, Elipe S, Oro LA, Lamata MP, Viguri F, Mir C, Cativiela C, Lopez-Ram de Viu MP. Synthesis, Characterization, Properties, and Asymmetric Catalytic Diels-Alder Reactions of Chiral-at-Metal Imino-Iridium(2+l) Complexes. Organometallics 1998;17:2986-2995.

23. Anderson C, Crespo M, Morris J, Tanski JM. Reactivity of cyclometallated platinum complexes with chiral ligands. $J$ Organomet Chem 2006;691:5635-5641.

24. Constable EC, Zhang G, Housecroft CE, Neuburger M, Zampese JA. T-Stacking and hydrogen bonding direct diastereoselectivity in one-pot syntheses of octahedral iron(2+) complexes. Chem Commun 2010;46:3077-3079.

25. Gu Z-G, Na J-J, Bao F-F, Xu X-X, Zhou W, Pang C-Y, Li Z. Synthesis, characterization, and DNA-binding of enantiomers of iron(2+) Schiff base complexes. Polyhedron 2013;51:186191.

26. Pescitelli G, Ludeke S, Chamayou A-C, Marolt M, Justus V, Gorecki M, Arrico L, Di Bari L, Islam MA, Gruber I, Enamullah M, Janiak C. Broad-Range Spectral Analysis for Chiral Metal Coordination Compounds: (Chiro)optical Superspectrum of Cobalt(2+) Complexes. Inorg Chem 2018;57:13397-13408.
27. Daneshmand P, Michalsky I, Aguiar PM, Schaper F. Configurationally flexible zinc complexes as catalysts for raclactide polymerisation. Dalton Trans 2018;47:16279-16291.

28. Li T-Y, Zheng Y-X, Zhou Y-H. Iridium(2+I) phosphorescent complexes with dual stereogenic centers: single crystal, electronic circular dichroism evidence and circularly polarized luminescence properties. Dalton Trans 2016:45:19234-19237.

29. Han W-K, Qin L-F, Pang C-Y, Cheng C-K, Zhu W, Li Z-H, Li $Z$, Ren X, Gu Z-G. Polymorphism of a chiral iron(2+) complex: spin-crossover and ferroelectric properties. Dalton Trans 2017;46:8004-8008.

30. Bin Jia, Junsheng Hao, Xuehong Wei, Hongbo Tong, Meisu Zhou, Diansheng Liu (2017) J.Organomet.Chem. ,831,11

31. Albert J, D'Andrea L, Granell J, Tavera R, Font-Bardia M, Solans X. Synthesis and reactivity towards carbon monoxide of an optically active endo five-membered orthocyclopalladated imine: X-ray molecular structure of trans-( $\mu$ $\mathrm{Cl})_{2}\left[\mathrm{Pd}\left(\mathrm{K}^{2}-\mathrm{C}, \mathrm{N}-(R)-\mathrm{C}_{6} \mathrm{H}_{4}-\mathrm{CH}=\mathrm{N}-\mathrm{CHMe}-\mathrm{Ph}\right)\right]_{2} . \quad J$ Organomet Chem 2007;692:3070-3080.

32. Fossey JS, Russell ML, Malik KMA, Richards CJ. Synthesis and crystal structures of the first $C_{2}$-symmetric bis-aldimine NCN-pincer complexes of platinum and palladium. $J$ Organomet Chem 2007;692:4843-4848.

33. Howson SE, Allan LEN, Chmel NP, Clarkson GJ, Deeth RJ, Faulkner AD, Simpson DH, Scott P. Origins of stereoselectivity in optically pure phenylethaniminopyridinetris-chelates $\mathrm{M}\left(\mathrm{NN}^{\prime}\right)_{3}{ }^{n+}(\mathrm{M}=\mathrm{Mn}$, Fe, Co, Ni and Zn). Dalton Trans 2011;40:10416-10433.

34. Gu Z-G, Pang C-Y, Qiu D, Zhang J, Huang J-L, Qin L-F, Sun A-Q, Li Z. Homochiral iron(2+) complexes based on imidazole Schiff-base ligands: Syntheses, structures, and spin-crossover properties. Inorg Chem Commun 2013;35:164-168.

35. Pait M, Shatruk M, Lengyel J, Gomez-Coca S, Bauza A, Frontera A, Bertolasi V, Ray D. Two types of nitrito support for $\mu_{4}$-oxido-bridged $\left[\mathrm{Cu}_{4}\right]$ complexes: synthesis, crystal structures, magnetic properties and DFT analysis. Dalton Trans 2015;44:6107-6117.

36. Zhang F-L, Tian L, Qin L-F, Chen J-Q, Li Z, Ren X, Gu Z-G. Chiral double helical silver complexes: subcomponent selfassembly and self-sorting. Polyhedron 2016;104:9-16.

37. Pastor MF, Whitehorne TJJ, Oguadinma PO, Schaper F. Zinc complexes of chiral ligands obtained from methylbenzylamine. Inorg Chem Commun 2011;14:17371741.

38. Qin L-F, Pang C-Y, Han W-K, Zhang F-L, Tian L, Gu Z-G, Ren X, Li Z. Spin crossover properties of enantiomers, coenantiomers, racemates, and co-racemates. Dalton Trans 2016;45:7340-7348.

39. Li L, Becker JM, Allan LEN, Clarkson GJ, Turner SS, Scott P. Structural and Electronic Modulation of Magnetic Properties in a Family of Chiral Iron Coordination Polymers. Inorg Chem 2011;50:5925-5935.

40. Han W-K, Li Z-H, Zhu W, Li T, Li Z, Ren X, Gu Z-G. Molecular isomerism induced $\mathrm{Fe}(2+)$ spin state difference based on the tautomerization of the 4(5)-methylimidazole group. Dalton Trans 2017;46:4218-4224.

41. Min KS, Park AH, Shin JW, Rowthu SR, Kim SK, Ryoo JJ. Synthesis and characterization of enantiopure copper(2+) complexes using chiral bidentate ligands. Dalton Trans 2010;39:8741-8747.

42. Mamula O, von Zelewsky A, Bark T, Stoeckli-Evans H, Neels A, Bernardinelli G. Predetermined chirality at metal centers of various coordination geometries: A chiral cleft ligand for tetrahedral (T-4), square-planar (SP-4), trigonal-bipyramidal (TB-5), square-pyramidal (SPY-5), and octahedral (OC-6) complexes. Chem Eur J 2000;6(19):3575-3585.

43. Knof U, von Zelewsky A. Predetermined chirality at metal centers. Angew Chem Int Ed Engl 1999;38:302-322.

44. Liu M, Zhang L, Wang T. Supramolecular Chirality in SelfAssembled Systems. Chem Rev 2015;115:7304-7397. 
45. Crusats J, Veintemillas-Verdaguer S, Ribo JM. Homochirality as a Consequence of Thermodynamic Equilibrium? Chem Eur J 2006;12:7776-7781.

46. Chen L-J, Yang H-B, Shionoya M. Chiral metallosupramolecular architectures. Chem Soc Rev 2017; 46:2555-2576.

47. Ohkita M, Lehn J-M, Baum G, Fenske D. Helicity Coding: Programmed Molecular Self- Organization of Achiral Nonbiological Strands into Multiturn Helical Superstructures: Synthesis and Characterization of Alternating PyridinePyrimidine Oligomers. Chem Eur J 1999;5:3471-3481.

48. Kramer R, Lehn J-M, De Cian A, Fischer J. Self-Assembly, Structure, and Spontaneous Resolution of a Trinuclear Triple Helix from an Oligobipyridine Ligand and $\mathrm{N}_{2}+{ }^{\prime}$ Ions. Angew Chem Int Ed Engl 1993;32:703-706

49. a) Dumitru F, Legrand Y-M, van der Lee A, Barboiu M. Constitutional self-sorting of homochiral supramolecular helical single-crystals from achiral components. Chem Commun 2009;19:2667-2669. b) Barboiu M, Dumitru F, Legrand Y-M, Petit E, van der Lee A. Self-sorting of equilibrating metallosupramolecular DCLs via constitutional crystallization. Chem Commun 2009;16:2192-2194. c) Dumitru F, Legrand Y-M, Barboiu M, van der Lee A. Weak intermolecular hydrogen and halogen interactions in an isomorphous halogen series of pseudoterpyridine $\mathrm{Zn}^{2+}$ complexes. Acta Cryst B 2013;69:43-54. d) Dumitru F, Legrand Y-M, Petit E, van der Lee A, Barboiu M. Self-sorting of metallosupramolecular DCLs via double-level exchange: amplification in solution and solid state modulation. Dalton Trans 2012;41(38):11860-11865. e) Dumitru F, Legrand YM, Barboiu M, Petit E, van der Lee A. Metallosupramolecular architectures of pseudoterpyridine-type ligands and $\mathrm{Zn}^{2+}$ metal ions. Cryst Growth Des 2009;9(6):2917-2921. f) Legrand Y-M, Dumitru F, van der Lee A, Barboiu M. Interpenetrated Constitutional Networks of Aromatic Metallosupramolecular Duplexes. Supramol Chem 2009;21(3-4):229-236. g) Dumitru F, Petit E, van der Lee A, Barboiu M. Homo- and heteroduplex complexes containing terpyridine-type ligands and $\mathrm{Zn}^{2+}$. Eur $J$ Inorg Chem 2005;21(9):4255-4262.

50. Vance AL, Alcock NW, Heppert JA, Busch DH. An Octahedral Template Based on a New Molecular Turn: Synthesis and Structure of a Model Complex and a Reactive, Diphenolic Ligand and Its Metal Complexes. Inorg Chem 1998:37:6912-6920.

51. CrysAlis ${ }^{\text {Pro }}$ Rigaku Oxford Diffraction Technologies, England 2012.

52. van der Lee, A. Charge flipping for routine structure solution. J Appl Crystallogr 2013;46:1306-1315.

53. Hooft RWW, Straver LH, Spek AL, Determination of absolute structure using Bayesian statistics on Bijvoet differences. J Appl Cryst 2008;41:96-103.
54. Palatinus L, Chapuis G. SUPERFLIP - a computer program for the solution of crystal structures by charge flipping in arbitrary dimensions. J Appl Crystallogr 2007;40:786-790.

55. a) Flack HD. On enantiomorph-polarity estimation. Acta Crystallogr A 1983;39:876-881. b) Flack HD, Bernardinelli G. Absolute structure and absolute configuration. Acta Crystallogr A 1999;55:908-915. c) Flack HD, Bernardinelli G. The use of X-ray crystallography to determine absolute configuration. Chirality 2008;20:681-690. d) Flack HD, Sadki M, Thompson AL, Watkin DJ. Acta Crystallogr A 2011;67:21-34. e) Parsons S, Flack HD, Wagner T. Use of intensity quotients and differences in absolute structure refinement. Acta Crystallogr B 2013;69:249-259. f) Hooft RWW, Straver LH, Spek A. Determination of absolute structure using Bayesian statistics on Bijvoet differences. $J$ Appl Cryst 2008;41:96-103.

56. Betteridge PW, Carruthers JR, Cooper RI, Prout K, Watkin DJ. CRYSTALS version 12: software for guided crystal structure analysis. J Appl Crystallogr 2003;36:1487-1487.

57. Cooper RI, Thompson AL, Watkin DJ. CRYSTALS enhancements: dealing with hydrogen atoms in refinement. J Appl Crystallogr 2010;46:1100-1107.

58. a) Somogyi L, Samu E, Huszthy P, Lazar A, Angyan JG, Surjan PR, Hollosi M. Circular dichroism of host-guest complexes of achiral pyridino- and phenazino-18-crown-6 ligands with the enantiomers of chiral aralkyl ammonium salts. Chirality 2001;13:109-117. b) Donnoli MI, Giorgio E, Superchi S, Rosini C. Circular dichroism spectra and absolute configuration of some aryl methyl sulfoxides. Org Biomol Chem 2003;1:3444-3449.

59. Llunell M, Casanova D, Cirera J, Alemany P, Alvarez S. SHAPE: Program for the Stereochemical Analysis of Molecular Fragments by Means of Continuous Shape Measures and Associated Tools, 2.1; University of Barcelona: Barcelona, 2013.

60. Alvarez S. Distortion Pathways of Transition Metal Coordination Polyhedra Induced by Chelating Topology. Chem Rev 2015;115:13447-13483.

61. Janiak C. A critical account on $\pi-\pi$ stacking in metal complexes with aromatic nitrogen-containing ligands. $J$ Chem Soc Dalton Trans 2000;21:3885-3896.

62. a) Langs DA. Three-dimensional structure at $0.86 \mathrm{~A}$ of the uncomplexed form of the transmembrane ion channel peptide gramicidin A. Science 1988;241:188-191. b) Cohen C. Why fibrous proteins are romantic. J Struct Biol 1998;122:3-16. c) Creager AN. The Life of a Virus: Tobacco Mosaic Virus as an Experimental Model, University of Chicago Press, 2002.

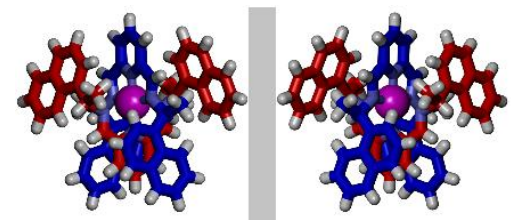

